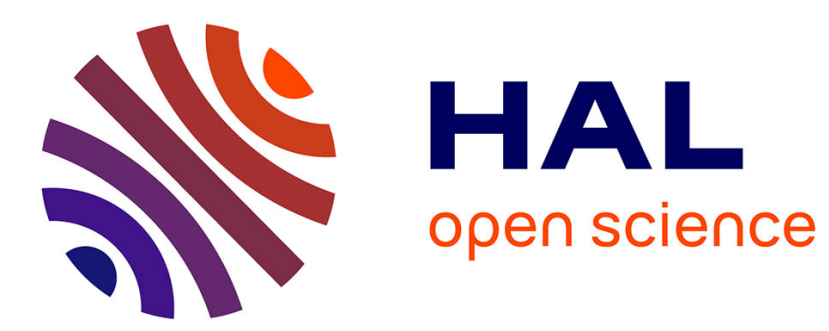

\title{
On the distance between separatrices for the discretized logistic differential equation
}

\author{
Hocine Sellama
}

\section{To cite this version:}

Hocine Sellama. On the distance between separatrices for the discretized logistic differential equation. 2007. hal-00143589v2

\section{HAL Id: hal-00143589 \\ https://hal.science/hal-00143589v2}

Preprint submitted on 12 Jun 2008

HAL is a multi-disciplinary open access archive for the deposit and dissemination of scientific research documents, whether they are published or not. The documents may come from teaching and research institutions in France or abroad, or from public or private research centers.
L'archive ouverte pluridisciplinaire HAL, est destinée au dépôt et à la diffusion de documents scientifiques de niveau recherche, publiés ou non, émanant des établissements d'enseignement et de recherche français ou étrangers, des laboratoires publics ou privés. 


\title{
On the distance between separatrices for the discretized logistic differential equation
}

\author{
Hocine Sellama \\ IRMA - UMR 7501 CNRS/ULP \\ 7 rue Ren Descartes - 67084 Strasbourg Cedex, France \\ email: sellama@math.u-strasbg.fr
}

In this paper, we consider the discretization

$$
y(t+\varepsilon)=y(t-\varepsilon)+2 \varepsilon\left(1-y(t)^{2}\right),
$$

$\varepsilon>0$ a small parameter, of the logistic differential equation $y^{\prime}=1-y^{2}$, which can also be seen as a discretization of the system

$$
\left\{\begin{array}{l}
y^{\prime}=2\left(1-v^{2}\right), \\
v^{\prime}=2\left(1-y^{2}\right) .
\end{array}\right.
$$

This system has two saddle points at $A=(1,1), B=(-1,-1)$ and there exist stable and unstable manifolds. We will show that the stable manifold $W_{s}^{+}$of the point $A=(1,1)$ and the unstable manifold $W_{i}^{-}$of the point $B=(-1,-1)$ for the discretization do not coincide. The vertical distance between these two manifolds is exponentially small but not zero, in particular we give an asymptotic estimate of this distance. For this purpose we will use a method adapted from the paper of Schäfke-Volkmer [5] using formal series and accurate estimates of the coefficients.

Keywords : Difference equation; Manifolds; Linear operator; Formal solution; Gevrey asymptotic; Quasi-solution

\section{Introduction}

We consider the logistic equation

$$
y^{\prime}=1-y(t)^{2},
$$

whose solutions are $y(t)=\tanh (t+c)$. The discretization of this equation by Nystrôm's method, which consists in replacing the derivative by the symmetrical difference, gives the recurrence :

$$
y_{n+1}=y_{n-1}+2 \varepsilon\left(1-y_{n}^{2}\right) .
$$




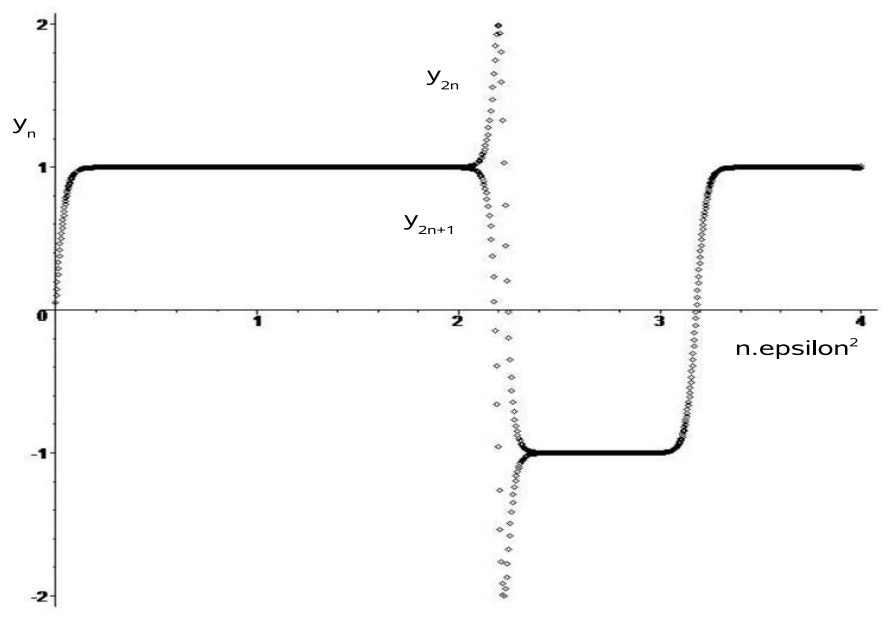

FIG. 1 - This figure represents $y_{n}$ as a function of $n \varepsilon^{2}$.

With the initial conditions $y_{0}=0, y_{1}=\varepsilon$, we calculate the discrete solution $y_{n}$ with 1600 iterates and $\varepsilon=\frac{1}{20}$ (see FIG.1). We observe that the discrete solution rejoins quickly the level $y=1$, it stays close to this point for a relatively long time before it leaves its neighborhood, then for the little while the even numbered points follow one curve and the odd numbered follow another curve, the two curves meet at the level $y=-1$, the discrete solution stays close to this point for a relatively long time, then it remakes the same cycle.

Now letting $u_{n}=y_{2 n}, \quad v_{n}=y_{2 n+1}$, we obtain a recurrence of first order in the plane

$$
\left(u_{n+1}, v_{n+1}\right)=\Phi\left(u_{n}, v_{n}\right)
$$

where the diffeomorphism $\Phi: \mathrm{IR}^{2} \mapsto \mathrm{IR}^{2}$ is defined by

$$
\begin{aligned}
& u_{1}=u+2 \varepsilon\left(1-v^{2}\right), \\
& v_{1}=v+2 \varepsilon\left(1-u_{1}^{2}\right) .
\end{aligned}
$$

This is a discretization of the following system of differential equations

$$
\left\{\begin{array}{l}
u^{\prime}=2\left(1-v^{2}\right), \\
v^{\prime}=2\left(1-u^{2}\right) .
\end{array}\right.
$$

We notice easily that the set $\mathfrak{E}=\left\{(u, v) \backslash(u-v)\left(u^{2}+u v+v^{2}-3\right)=0\right\}$ is an invariant set for this system. The system (1.4) has two saddle points in $A=(1,1)$ 
and $B=(-1,-1)$ and corresponding stable and unstable manifolds lie on its invariant set $\mathfrak{E}$.

The stable manifold at $A$ and unstable manifolds at $B$ are part of set $\{(u, v) \backslash u=$ $v\}$, where as the unstable manifold at $A$ and stable manifolds at $B$ are part of set $\left\{(u, v) \backslash u^{2}+u v+v^{2}=3\right\}$. The stable manifold of $A$ coincides with the unstable manifold of $B$ (See FIG.2).

For the discretized equation (1.3), these manifolds still exist [2], let $W_{s}^{+}, W_{i}^{-}$ denote the stable manifold at $A$ and the unstable manifold at $B$ respectively, $W_{i}^{+}$ and $W_{s}^{-}$unstable manifold at $A$ and stable manifold at $B$ respectively, $W_{s}^{+}$and $W_{i}^{-}$do not coincide an more (See FIG.3) as we want to show.

In the paper [2], after introducing the notion of length of the first level $l_{1}(\varepsilon)=$ $2 \varepsilon n_{1}(\varepsilon)$, where $n_{1}(\varepsilon)=\inf \left\{n \in \operatorname{IN} \backslash y_{2 n}(\varepsilon)+y_{2 n+1}(\varepsilon)<0\right\}$, Fruchard-Schäfke had shown that there exist a postive constant $K$ such that

$$
\begin{aligned}
& \Delta(\varepsilon) \leq \exp \left(-\frac{\pi^{2}+o(1)}{2 \varepsilon}\right), \quad \text { as } \varepsilon \searrow 0, \\
& l_{1}(\varepsilon) \geq \frac{\pi^{2}}{4 \varepsilon}+o(1),
\end{aligned}
$$

where $\Delta(\varepsilon)$ denote the distance between the sets $W_{s}^{+} \cap S$ and $W_{i}^{-} \cap S$ in the sense of Hausdorff and $S=\{(u, v) \backslash-1 \leq u+v \leq 1\}$.

With the initial condition $y_{0}=0, y_{1}=\varepsilon$, the length of the first level satisfies $l_{1}(\varepsilon)=-\frac{1}{2}(1+o(1)) \log (\Delta(\varepsilon))$ as $\varepsilon \searrow 0[2]$. They also showed that there are two families of entire functions $y_{\varepsilon}^{ \pm}: \mathbb{C} \longmapsto \mathbb{C}$, solutions of the difference equation

$$
y(t+\varepsilon)=y(t-\varepsilon)+2 \varepsilon\left(1-y(t)^{2}\right),
$$

and the functions $t \mapsto\left(y_{\varepsilon}^{+}(t), y_{\varepsilon}^{+}(t+\varepsilon)\right), t \mapsto\left(y_{\varepsilon}^{-}(t), y_{\varepsilon}^{-}(t+\varepsilon)\right)$ provide parametrization $w_{s}^{+}(t)$ of $W_{s}^{+}$for $t \in\left[-1, \infty\left[\right.\right.$ respectively $w_{i}^{-}(t)$ of $W_{i}^{-}$for $\left.\left.t \in\right]-\infty, 1\right]$.

In this work we will prove

Theorem 1.1. There exist a constant $\alpha$ with $1.2641497 \leq \alpha \leq 1.2641509$ and $\varepsilon_{0}>0$ such that for $0<\varepsilon<\varepsilon_{0}$

$$
\operatorname{Dist}_{\varepsilon}\left(w_{s}^{+}(t), W_{i}^{-}\right)=\frac{4 \pi \alpha \cos \left(\frac{\pi}{\varepsilon} t+\pi\right)}{\varepsilon^{3}\left(1-\tanh (t)^{2}\right)} e^{-\frac{\pi^{2}}{2 \varepsilon}}+\mathcal{O}\left(\frac{1}{\varepsilon^{2}} e^{-\frac{\pi^{2}}{2 \varepsilon}}\right), \quad \text { as } \varepsilon \searrow 0,
$$

where Dist is $_{\text {in }}$ defined as the vertical distance between the stable and unstable manifolds.

In order to show the theorem 1.1, we start with the construction of a formal power series solution in $d$ whose coefficients are polynomials in $u=\tanh (d t / \varepsilon)$, afterwards we will give asymptotic approximations of these coefficients using appropriate norms on the spaces of polynomials. The next step is to construct a quasi-solution i.e. a function that satisfies the equation (1.5) except for an exponentially small error, then we show that this quasi-solution and the exact solution of equation (1.5) are exponentially close. 


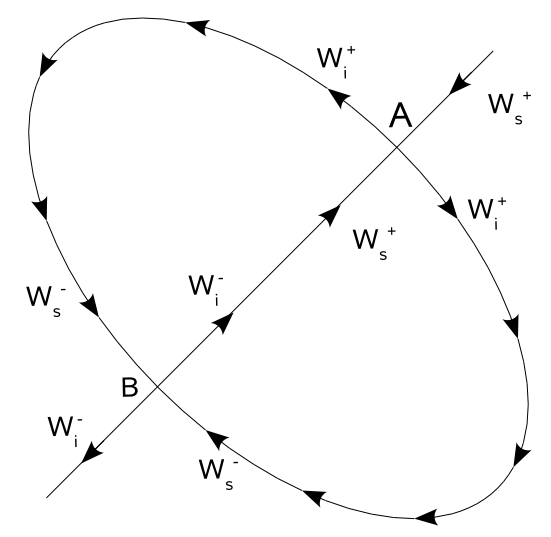

FIG. 2 - The stable and unstable manifolds for the logistic differential equation.

Finally we give an asymptotic estimate of the distance between the stable manifold of $A$ and the unstable manifold of $B$ and we show that this distance is exponentially small but not zero, thus completing the proof of the theorem 1.1.

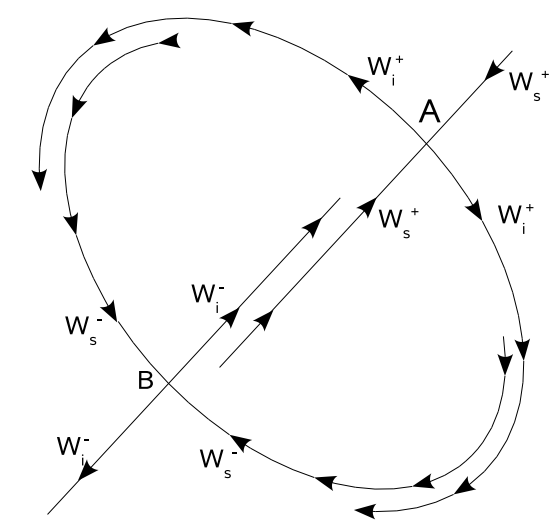

FIG. 3 - The stable and unstable manifolds for the difference equation.

\section{Formal solutions}

We consider the difference equation

$$
y(t+\varepsilon)=y(t-\varepsilon)+2 \varepsilon\left(1-y(t)^{2}\right),
$$

where $\varepsilon>0$ is the discretization step and $y(t) \rightarrow 1$ as $t \rightarrow+\infty$. Our first aim is to transform (2.1) in such a way that the new equation admits a formal solutions whose coefficients are polynomials. We define $u$ and $A(d, u)$ such that

$$
u:=\tanh \left(\frac{d}{\varepsilon} t\right),
$$




$$
y_{\varepsilon}(t)=A(d, u),
$$

where $d:=\sum_{n=1}^{+\infty} d_{n} \varepsilon^{n}$ is a series to be determined. When these variables are substituted in (2.1), the following equation satisfied by $A(d, u)$ is obtained :

$$
A\left(d, T^{+}(d, u)\right)-A\left(d, T^{-}(d, u)\right)=f(\varepsilon, A(d, u)),
$$

where

$$
\begin{gathered}
f(\varepsilon, x):=2 \varepsilon\left(1-x^{2}\right), \\
T^{+}:=T^{+}(d, u)=\frac{u+\tanh (d)}{1+u \tanh (d)}, \\
T^{-}:=T^{-}(d, u)=\frac{u-\tanh (d)}{1-u \tanh (d)} .
\end{gathered}
$$

For small $\varepsilon$ one can construct a formal expansion in powers of $\varepsilon^{2}$ of the form $\sum_{n=0}^{\infty} A_{2 n+1}(u) d^{2 n}$, where $A_{2 n+1}$ are polynomials which satisfy $A_{1}(u)=u$ and $A_{2 n+1}( \pm 1)=0$, for $n \geq 1$.

The existence of a such formal solution is only possible if $d$ and $\varepsilon$ are coupled in a very special way. Indeed, suppose that there exists a such formal power series solution. We differentiate (2.4) with respect to $\mathrm{u}$ and obtain at $u=1$

$$
\frac{\partial A}{\partial u}(d, 1) \cdot \lim _{u \rightarrow 1} \frac{T^{+}(d, u)-1}{u-1}-\frac{\partial A}{\partial u}(d, 1) \cdot \lim _{u \rightarrow 1} \frac{T^{-}(d, u)-1}{u-1}=\frac{\partial f}{\partial x}(\varepsilon, 1) \cdot \frac{\partial A}{\partial u}(d, 1) .
$$

Because $A_{2 n+1}(1)=0$ for $n \geq 1$ implies $A(d, 1)=1$. Thus with $\frac{\partial f}{\partial x}(\varepsilon, 1)=-4 \varepsilon$ and $\frac{\partial A}{\partial u}(d, 1)=1+O(d) \neq 0$, the above equation implies

$$
\varepsilon=\frac{\tanh (d)}{1-\tanh (d)^{2}}=\frac{1}{2} \sinh (2 d) .
$$

Theorem 2.1. Let $\varepsilon$ and $d$ be coupled by $\varepsilon=\frac{1}{2} \sinh (2 d)$. Then (2.4) has a formal power series solution that can be written in the form

$$
A(d, u)=u+\sum_{n=1}^{\infty} A_{2 n+1}(u) d^{2 n},
$$

where $A_{2 n+1}(u)$ are odd polynomial and $A_{2 n+1}(1)=A_{2 n+1}(-1)=0$ for all $n$.

Proof. We will use the Induction Principle for even $\mathrm{n}$ to show that there exist unique odd polynomials $A_{1}, A_{3}, A_{5} \ldots A_{n+1}$ such that

$$
Z(d, u)=\sum_{\substack{k=0 \\ k \text { even }}}^{n} A_{k+1} d^{k},
$$


satisfy

$$
Z\left(d, T^{+}(d, u)\right)-Z\left(d, T^{-}(d, u)\right)=f(\varepsilon, Z(d, u)) \bmod d^{n+2} .
$$

For $n=0$, we put $A_{1}(u)=u$ and $Z(d, u)=u$ and obtain

$$
Z\left(d, T^{+}(d, u)\right)-Z\left(d, T^{-}(d, u)\right)=T^{+}(d, u)-T^{-}(d, u)=\left(2-2 u^{2}\right) d+\mathcal{O}\left(d^{3}\right) .
$$

and

$$
f(\varepsilon, Z(d, u))=2 \varepsilon\left(1-u^{2}\right)=\left(2-2 u^{2}\right) d+\mathcal{O}\left(d^{3}\right) .
$$

This gives

$$
Z\left(d, T^{+}(d, u)\right)-Z\left(d, T^{-}(d, u)\right)=f(\varepsilon, Z(d, u)) \bmod d^{2} .
$$

Now suppose that for some even $n$ already $A_{1}, A_{3}, A_{5} \ldots A_{n+1}$ have been found with the above properties. We have to construct $A_{n+3}$. First, we show that $Z(d, u)$ satisfies (2.11) even modulo $d^{n+3}$. To this purpose, let

$$
Z\left(d, T^{+}\right)-Z\left(d, T^{-}\right)=f(\varepsilon, Z(d, u))+R_{n+2}(u) d^{n+2}+\mathcal{O}\left(d^{n+3}\right)
$$

we replace $d$ by $-d$. Using that $Z$ is even and $\varepsilon$ is odd in $d$, we obtain

$$
Z\left(d, T^{-}\right)-Z\left(d, T^{+}\right)=-2 \varepsilon\left(1-Z(d, u)^{2}\right)+R_{n+2}(u) d^{n+2}+\mathcal{O}\left(d^{n+3}\right),
$$

this gives

$$
Z\left(d, T^{+}\right)-Z\left(d, T^{-}\right)=2 \varepsilon(d)\left(1-Z(d, u)^{2}\right)-R_{n+2}(u)(d)^{n+2}+\mathcal{O}\left(d^{n+3}\right) .
$$

With (2.12) this implies $R_{n+2}(u)=0$ and consequently

$$
Z\left(d, T^{+}\right)-Z\left(d, T^{-}\right)=f(\varepsilon, Z(d, u))+R_{n+3}(u) d^{n+3}+\mathcal{O}\left(d^{n+4}\right) .
$$

We want to construct $A_{n+3}(u)$ such that

$$
\widetilde{Z}\left(d, T^{+}\right)-\widetilde{Z}\left(d, T^{-}\right)=f(\varepsilon, \widetilde{Z}(d, u))+\mathcal{O}\left(d^{n+4}\right),
$$

if we put

$$
\widetilde{Z}(d, u)=Z(d, u)+A_{n+3}(u) d^{n+2} .
$$

To this purpose we use again Taylor expansion

$$
\begin{array}{rlr}
f(\varepsilon, \widetilde{Z}(d, u)) & =f(\varepsilon, Z(d, u))-4 u A_{n+3}(u) d^{n+3}+\quad \mathcal{O}\left(d^{n+4}\right), \\
\widetilde{Z}\left(d, T^{+}(d, u)\right) & =Z(d, u)+A_{n+3}(u) d^{n+2}+\left(1-u^{2}\right) \frac{\partial A_{n+3}}{\partial u}(u) d^{n+3}+\mathcal{O}\left(d^{n+4}\right), \\
\widetilde{Z}\left(d, T^{-}(d, u)\right) & =Z(d, u)+A_{n+3}(u) d^{n+2}-\left(1-u^{2}\right) \frac{\partial A_{n+3}}{\partial u}(u) d^{n+3}+\mathcal{O}\left(d^{n+4}\right) .
\end{array}
$$


With (2.13), this gives

$$
\widetilde{Z}\left(d, T^{+}\right)-\widetilde{Z}\left(d, T^{-}\right)=f(\varepsilon, \widetilde{Z}(d, u))+\widetilde{R}_{n+3}(u) d^{n+3}+\mathcal{O}\left(d^{n+4}\right),
$$

where

$$
\widetilde{R}_{n+3}(u)=2\left(1-u^{2}\right) \frac{\partial A_{n+3}}{\partial u}(u)+4 u A_{n+3}(u)+R_{n+3}(u) .
$$

We see that (2.14) is satisfied if only if

$$
2\left(1-u^{2}\right) \frac{\partial A_{n+3}}{\partial u}(u)+4 u A_{n+3}(u)+R_{n+3}(u)=0 .
$$

This equation has a unique odd solution which is given by

$$
A_{n+3}(u)=-\left(1-u^{2}\right) \int_{0}^{u} \frac{R_{n+3}(t)}{2\left(1-t^{2}\right)^{2}} d t .
$$

We can prove that this solution is polynomial. For that, it is necessary that $R_{n+3}(u)$ and $R_{n+3}^{\prime}(u)$ vanish into 1 and -1 . Indeed, if we take (2.13) with $u=1$, we obtain

$$
R_{n+3}(1) d^{n+3}=Z\left(d, T^{+}(d, 1)\right)-Z\left(d, T^{-}(d, 1)\right)-f(\varepsilon, Z(d, 1))+\mathcal{O}\left(d^{n+4}\right) .
$$

Since, $T^{+}(d, 1)=T^{-}(d, 1)=Z(d, 1)=1$ and $f(\varepsilon, 1)=0$, we obtain

$$
R_{n+3}(1) d^{n+3}=\mathcal{O}\left(d^{n+4}\right),
$$

therefore $R_{n+3}(1)=0$. In order to show that $R_{n+3}^{\prime}(1)=0$, we derive formally (2.13) and replace $u$ by 1 . Using $T^{+}(d, 1)=T^{-}(d, 1)=Z(d, 1)=1$ and $\frac{\partial f}{\partial u}(\varepsilon, 1)=$ $-4 \varepsilon$, we obtain

$$
R_{n+3}^{\prime}(1) d^{n+3}=\frac{\partial Z}{\partial u}(d, 1)\left(\frac{\partial T^{+}}{\partial u}(d, 1)-\frac{\partial T^{-}}{\partial u}(d, 1)+4 \varepsilon\right)+\mathcal{O}\left(d^{n+4}\right) .
$$

By choice of $d$, cf (2.8). Thus we have $\frac{\partial T^{+}}{\partial u}(d, 1)-\frac{\partial T^{-}}{\partial u}(d, 1)=-2 \sinh (2 d)$. with $(2,8)$ we obtain

$$
R_{n+3}^{\prime}(1) d^{n+3}=\mathcal{O}\left(d^{n+4}\right),
$$

therefore $R_{n+3}^{\prime}(1)=0$. As formula (2.13) shows that $R_{n+3}(u)$ is odd, then $R_{n+3}(-1)=$ $R_{n+3}^{\prime}(-1)=0$.

The first polynomials $A_{n+1}(u)$ with even $n$ are given by

\begin{tabular}{|c|c|c|c|c|}
\hline$n$ & 0 & 2 & 4 & 6 \\
\hline$A_{n+1}(u)$ & $u$ & $u-u^{3}$ & $\left(1-u^{2}\right)\left(\frac{4}{3} u-\frac{10}{3} u^{3}\right)$ & $\left(1-u^{2}\right)\left(\frac{62}{3} u^{5}-\frac{190}{9} u^{3}+\frac{182}{45} u\right)$ \\
\hline
\end{tabular}


We introduce the operators $\mathcal{C}_{2}, \mathcal{C}, \mathcal{S}_{2}, \mathcal{S}$ defined by

$$
\begin{aligned}
& \mathcal{C}(Z)(d, u)=\frac{1}{2}\left(Z\left(d, T^{+\frac{1}{2}}\right)+Z\left(d, T^{-\frac{1}{2}}\right)\right), \\
& \mathcal{S}(Z)(d, u)=\frac{1}{2}\left(Z\left(d, T^{+\frac{1}{2}}\right)-Z\left(d, T^{-\frac{1}{2}}\right)\right), \\
& \mathcal{C}_{2}(Z)(d, u)=\frac{1}{2}\left(Z\left(d, T^{+}\right)+Z\left(d, T^{-}\right)\right), \\
& \mathcal{S}_{2}(Z)(d, u)=\frac{1}{2}\left(Z\left(d, T^{+}\right)-Z\left(d, T^{-}\right)\right) .
\end{aligned}
$$

where $T^{+\frac{1}{2}}=T^{+}\left(\frac{d}{2}, u\right), T^{-\frac{1}{2}}=T^{-}\left(\frac{d}{2}, u\right)$ and $Z(d, u)$ is a formal power series of $\mathrm{d}$ whose coefficients are polynomials. We rewrite equation (2.4) as

$$
\mathcal{S}_{2}(A)(d, u)=\varepsilon\left(1-A(d, u)^{2}\right) .
$$

\section{Norms for polynomials and basis}

In the sequel we denote :

- $\mathcal{P}$ the set of all polynomial whose coefficents are complex,

- $\mathcal{P}_{n}$ the spaces of all polynomials of degree less than or equal to $\mathrm{n}$,

- $\mathcal{Q}:=\left\{Q(d, u)=\sum_{n=0}^{\infty} Q_{n}(u) d^{n}\right.$, where $Q_{n}(u) \in \mathcal{P}_{n}$, for all $\left.n \in \mathbb{N}\right\}$.

Proposition 3.1. [5] If we define the sequence of the polynomial functions $\tau_{n}(u)$ by $\tau_{0}(u)=1, \tau_{1}(u)=u, \tau_{n+1}(u)=\frac{1}{n} D \tau_{n}(u)$, where the operator $D$ is defined by $D:=\left(1-u^{2}\right) \frac{\partial}{\partial u}$, we have

$$
\begin{aligned}
& \text { 1. } T^{+}(d, u)=\sum_{n=0}^{\infty} \tau_{n+1}(u) d^{n}, \\
& \text { 2. } \tau_{n}(u) \text { has exactly degree } n, \\
& \text { 3. } \tau_{n}(\tanh (z))=\frac{1}{(n-1) !}\left(\frac{d}{d z}\right)^{n-1}(\tanh (z)) .
\end{aligned}
$$

Definition 3.2. Let $p \in \mathcal{P}_{n}$. As $\tau_{0}(u), \tau_{1}(u), \ldots, \tau_{n}(u)$ form a basis of $\mathcal{P}_{n}$, we can write $p$ as

$$
p=\sum_{k=0}^{n} a_{k} \tau_{k}(u)
$$

Then we define the norm

$$
\|p\|_{n}=\sum_{i=0}^{n} a_{i}\left(\frac{\pi}{2}\right)^{n-i}
$$

Theorem 3.3. 5 Let $n, m$ be positive integers and $p \in \mathcal{P}_{n}, q \in \mathcal{P}_{m}$. The norms \|\|$_{n}$ of the above definition have the following property: 
1. $\|D p\|_{n+1} \leq n\|p\|_{n}$.

2. If $p$ odd we have $\|p\|_{n} \leq\|D p\|_{n+1}$.

3. There exists a constant $M_{1}$ such that $\|p q\|_{n+m} \leq M_{1}\|p\|_{n} \mid q \|_{m}$.

4. There is a constant $M_{2}$ such that $|p(u)| \leq M_{2}\left(\frac{2}{\pi}\right)^{n}\|p\|_{n}(-1 \leq u \leq 1)$.

5. There is a constant $M_{3}$ such that for all $n>1$ with $p(-1)=p(-1)=0$,

$$
\left\|\frac{p}{\tau_{2}}\right\|_{n-2} \leq M_{3}\|p\|_{n}
$$

\section{Operators}

In this section we will use definitions and results adapted from [0] by replacing $\frac{\pi}{2}$ by $\pi$.

Definition 4.1. Let $f$ be formal power series of $z$ whose coefficients are complex. We define a linear operator $f(d D)$ on $\mathcal{Q}$ by

$$
f(d D) Q=\sum_{n=0}^{\infty}\left(\sum_{i=0}^{n} f_{i} D^{i} Q_{n-i}(u)\right) d^{n},
$$

Where $f(z)=\sum_{i=0}^{\infty} f_{i} z^{i}$ and $Q=\sum_{n=0}^{\infty} Q_{n}(u) d^{n} \in \mathcal{Q}$.

By the above definition and (1) of the proposition 3.1, we can show that

$$
Q\left(d, T^{+}(\theta d, u)\right)=(\exp (\theta d D) Q)(d, u), \quad \text { for } \quad Q \in \mathcal{Q} \text { and all } \theta \in \mathbb{C} .
$$

With (2.22) this implies

$$
\begin{aligned}
& \mathcal{C}(Q)(d, u)=\cosh \left(\frac{d}{2} D\right) Q, \mathcal{S}(Q)(d, u)=\sinh \left(\frac{d}{2} D\right) Q \\
& \mathcal{C}_{2}(Q)(d, u)=\cosh (d D) Q, \mathcal{S}_{2}(Q)(d, u)=\sinh (d D) Q
\end{aligned}
$$

for polynomial series $Q$ in $\mathcal{Q}$. We denote $\|Q\|_{n}=\left\|Q_{n}\right\|_{n}$.

Theorem 4.2. . Let $f(z)$ be formal power series having a radius of convergence greater than $\pi$ and let $k$ be a positive integer. There is a constant $K$ such that : If $Q$ is a polynomial series having the following property

$$
\|Q\|_{n} \leq \begin{cases}0 & \text { for } n<k \\ M(n-k) ! \pi^{-n} & \text { for } n \geq k\end{cases}
$$

where $M$ independent of $n$ and $Q \in \mathcal{Q}$ then the polynomial series $f(d D) Q$ satisfies

$$
\|f(d D) Q\|_{n} \leq \begin{cases}0 & \text { for } n<k \\ M K(n-k) ! \pi^{-n} & \text { for } n \geq k\end{cases}
$$


We define the operator $\mathcal{C}^{-1}$ by $\mathcal{C}^{-1}=g(d D)$, where $g(z)=\frac{1}{\cosh (z / 2)}$

Theorem 4.3. There exists a positive constant $K$ such that, if $Q$ is a polynomial series such that $Q_{n}$ odd, $\|Q\|_{n}=0$ for $n<k$ for some positive integer $k$ and

$$
\|d D Q\|_{n} \leq M(n-k) ! \pi^{-n} \text { for } n \geq k
$$

where $M$ independent of $n$ and $Q \in \mathcal{Q}$, the polynomial series $\mathcal{C}^{-1}(Q)$ satisfies

$$
\left\|\mathcal{C}^{-1}(Q)\right\|_{n} \leq M K \pi^{-n} \begin{cases}n ! & \text { for } k=1 \\ (n-1) ! \log (n) & \text { for } k=2 \\ (n-1) ! & \text { for } k \geq 3\end{cases}
$$

Theorem 4.4. We consider a polynomial series

$$
Q_{\alpha}(d, u)=\sum_{\substack{n=1 \\ n \text { odd }}}^{\infty} \alpha_{n}(n-1) !\left(\frac{i}{\pi}\right)^{n-1} \tau_{n}(u) d^{n}
$$

where $\alpha_{n}=\mathcal{O}\left(n^{-k}\right)$ as $n \rightarrow \infty$ with some integer $k \geq 2$. Let $\alpha:=\frac{1}{\pi} \sum_{n=1}^{\infty} \alpha_{n}$. then the coefficients $\left\{\mathcal{C}^{-1}\left(Q_{\alpha}\right)\right\}_{n}$ of $\mathcal{C}^{-1}\left(Q_{\alpha}\right)$ satisfy

$$
\left\|\left\{\mathcal{C}^{-1}\left(Q_{\alpha}\right)\right\}_{n}-\alpha(n-1) !\left(\frac{i}{\pi}\right)^{n-1} \tau_{n}\right\|_{n}=\mathcal{O}\left((n-k) ! \pi^{-n}\right),
$$

as $n \rightarrow \infty$ for odd $n$.

Theorem 4.5. Let $k, l, p, q$ be positive integer with $p \geq k$ and $q \geq l$. Define $m$ as the minimum of $k+q$ and $l+p$. Then there is a constant $K$ with the following property :

If $P$ and $Q$ are polynomial series such that $\|P\|_{n}=0$ for $n<p,\|Q\|_{n}=0$ for $n<q$ and

$$
\begin{aligned}
& \|P\|_{n} \leq M_{1}(n-k) ! \pi^{-n}, \quad \text { for } n \geq k \\
& \|Q\|_{n} \leq M_{2}(n-l) ! \pi^{-n}, \quad \text { for } n \geq l .
\end{aligned}
$$

Then

$$
\|P Q\|_{n} \leq K M_{1} M_{2}(n-m) ! \pi^{-n}, \text { for } n \geq p+q .
$$

Theorem 4.6. Let $Q_{1}(d, u)$ be a convergent polynomial series which is even with respect to both variables and has constant term 1.

Let $Q_{2}(d, u)=d^{2}\left(1-u^{2}\right) Q_{1}(d, u)$ and $P(d, u)=\mathcal{S}\left(Q_{2}\right) / \mathcal{C}\left(Q_{2}\right)$. Consider the linear operator defined by

$$
\mathcal{L}(Q)=\mathcal{S}(Q)-P(d, u) \cdot \mathcal{C}(Q), \quad Q \in \mathcal{Q} .
$$


Then, there exist a constant $K$ with the following property. If $Q$ is an odd polynomial series with odd coefficients $Q_{n}(u)$ satisfying $Q_{n}(1)=0$ for all $n,\|\mathcal{L}(Q)\|_{n}=0$ for $n<6$ and

$$
\|\mathcal{L}(Q)\|_{n} \leq M(n-6) ! \pi^{-n} \text { for } n \geq 6
$$

then also

$$
\|d D Q\|_{n} \leq K M(n-6) ! \pi^{-n} \text { for } n \geq 6 .
$$

\section{Asymptotic approximation of the coefficients of the formal solution}

The objective in this section is to construct an asymptotic aproximation of the coefficents of the formal solution (2.9). It will turn out to be convenient to consider the new series $B(d, u)=\varepsilon A(d, u)$,

$$
B(d, u)=\varepsilon A(d, u)=\sum_{\substack{n=2 \\ n \text { even }}}^{\infty} A_{n+1}(u) \varepsilon d^{n}+\varepsilon u,
$$

this gives

$$
B(d, u)=\sum_{\substack{n=3 \\ n \text { odd }}}^{\infty} B_{n}(u) d^{n}+\varepsilon u,
$$

where $B_{n}(u)$ are odd polynomials. Furthermore we have $B_{n}(u) \in \mathcal{P}_{n}$ and $B_{n}(1)=$ $B_{n}(-1)=0$ for all $n$. The new equation for $B(d, u)$ is

$$
\mathcal{S}_{2}(B)(d, u)=\varepsilon^{2}-B(d, u)^{2} .
$$

We saw in section 2 that the series $A$ was a formal solution of the starting equation. For the moment, nothing is known about the norms of its coefficients, but we will show that this series is Gevrey-1, more precisely $\|A\|_{n}=O\left(n ! \pi^{-n}\right)$. This enables us thereafter to construct a quasi-solution. To this purpose, we will prepare the equation (5.1) so that we can construct a recurrence.

We start with the decompostion of series $B(d, u)$ in the form :

$$
B=U+F, \text { where } \quad U=\varepsilon u+\left(u-u^{3}\right) d^{3}+\left(\frac{10}{3} u^{5}-\frac{16}{3} u^{3}+2 u\right) d^{5} .
$$

Next, we define

$$
\begin{aligned}
G: & =Q F, \\
J: & =\frac{\mathcal{C}(G)}{Q_{1}} .
\end{aligned}
$$


where

$$
\begin{aligned}
Q(d, u): & =\left(1-u^{2}\right) d^{2}+\left(u^{4}-u^{2}\right) d^{4}-\left(\frac{13}{6} u^{6}-\frac{7}{2} u^{4}+\frac{4}{3} u^{2}\right) d^{6} \\
& +\left(\frac{47}{6} u^{8}-\frac{31}{6} u^{6}+\frac{58}{15} u^{4}-\frac{104}{45} u^{2}+1\right) d^{8} \\
Q_{1}(d, u) & =\left(1-u^{2}\right) d^{2}+\frac{3}{2}\left(u^{2}-u^{4}\right) d^{4}
\end{aligned}
$$

The choice of $Q$ and $Q_{1}$ and by using the properties of the operators $\mathcal{S}, \mathcal{C}$, we will be able to rewrite the equation (5.1) in the form :

$$
\begin{aligned}
e_{0}(d, u) \mathcal{L}(J)= & e_{1}(d, u) \mathcal{C}(J)+e_{2}(d, u) F+e_{3}(d, u) F^{2} \\
& +e_{4}(d, u) \mathcal{C}_{2}(F)+e_{5}(d, u)
\end{aligned}
$$

where $\mathcal{L}$ is the operator defined in (4.3) and $e_{i}(d, u), i=0, \ldots, 5$ are convenient known convergent series in $d$ and $u$ which will be thereafter given.

The left hand side of this equation is series with leading term 1 multiplied by the invertible operator $\mathcal{L}$ applied to the series $J$. The right hand side is an expression of $F$ and $J$ multiplied by known convergent series $e_{i}(d, u), i=1, \ldots, 5$.

$U, Q, Q_{1}$ were chosen so that the series $e_{i}(d, u), i=0 . .5$ are of a rather large order in $d$, this makes the second term smaller than the right hand side. This property will be useful to construct a recurrence on $n$ and to reverse then the operators $\mathcal{L}, \mathcal{C}$, which makes possible to estimate the coefficients of series $\mathcal{C}(G)$ and finally the coefficients of the formal solution of the equation (5.1).

More precisely, we insert (5.2) into the equation (5.1) and find

$$
\mathcal{S}_{2}(F)=-F^{2}-2 U F+\varepsilon^{2}-U^{2}-\mathcal{S}_{2}(U) .
$$

We define $\mathcal{X}$ by

$$
\mathcal{X}:=V(d, u) \cdot \mathcal{S C}(G)+W(d, u) \cdot \mathcal{C}^{2}(G)
$$

where

$$
\begin{aligned}
V(d, u): & =1+2 u^{2} d^{2}-\left(\frac{7}{3} u^{4}-3 u^{2}\right) d^{4}-\left(\frac{31}{3} u^{6}-\frac{221}{9} u^{4}+\frac{253}{15} u^{2}-\frac{41}{15}\right) d^{6}, \\
W(d, u): & =2 \varepsilon u .
\end{aligned}
$$

Then, because of $G=Q F$, we have $\mathcal{X}(G)=V \cdot \mathcal{S C}(F Q)+W \cdot \mathcal{C}^{2}(F Q)$. Using the formulas

$$
\begin{aligned}
& \mathcal{C}_{2}=2 \mathcal{C}^{2}-I d, \\
& \mathcal{S}_{2}=2 \mathcal{S C}, \\
& \mathcal{S}_{2}(F Q)=\mathcal{S}_{2}(F) \mathcal{C}_{2}(Q)+\mathcal{S}_{2}(Q) \mathcal{C}_{2}(F), \\
& \mathcal{C}_{2}(F Q)=\mathcal{C}_{2}(F) \mathcal{C}_{2}(Q)+\mathcal{S}_{2}(Q) \mathcal{S}_{2}(F) .
\end{aligned}
$$


We obtain

$$
\begin{aligned}
\mathcal{X}= & \frac{1}{2} V \cdot \mathcal{S}_{2}(F Q)+\frac{1}{2} W \cdot\left(\mathcal{C}_{2}(F Q)+Q F\right) \\
= & \frac{1}{2}\left(V \mathcal{C}_{2}(Q)+W \mathcal{S}_{2}(Q)\right) \mathcal{S}_{2}(F)+\frac{1}{2}\left(V \mathcal{S}_{2}(Q)+W \mathcal{C}_{2}(Q)\right) \mathcal{C}_{2}(F) \\
& +\frac{1}{2} W Q \cdot F .
\end{aligned}
$$

With (5.5) this implies

$$
\mathcal{X}=\frac{1}{2} W_{1} F+\frac{1}{2} W_{2} \mathcal{C}_{2}(F)-\frac{1}{2}\left(V \mathcal{C}_{2}(Q)+W \mathcal{S}_{2}(Q)\right) F^{2}+\mu(\varepsilon, u),
$$

where

$$
\begin{aligned}
& W_{1}=-2 U\left(V \mathcal{C}_{2}(Q)+W \mathcal{S}_{2}(Q)\right)+W Q \\
& W_{2}=V \mathcal{S}_{2}(Q)+W \mathcal{C}_{2}(Q), \\
& \mu(\varepsilon, u)=\frac{1}{2}\left(V \mathcal{C}_{2}(Q)+W \mathcal{S}_{2}(Q)\right)\left(\varepsilon^{2}-U^{2}-\mathcal{S}_{2}(U)\right) .
\end{aligned}
$$

The lowest power of $d$ in $W_{1}$ and $W_{2}$ is the eleventh $W_{1}=\mathcal{O}\left(d^{11}\right)=W_{2}$, $\mu(\varepsilon, u)$ is analytic. On the other hand $\mathcal{C}(G)=Q_{1} J$, this implies

$$
\mathcal{X}(G)=V \cdot \mathcal{S}\left(Q_{1} J\right)+W \cdot \mathcal{C}\left(Q_{1} J\right) .
$$

If we use the product formulas

$$
\begin{aligned}
& \mathcal{S}\left(Q_{1} J\right)=\mathcal{S}(J) \mathcal{C}\left(Q_{1}\right)+\mathcal{S}\left(Q_{1}\right) \mathcal{C}(J), \\
& \mathcal{C}\left(Q_{1} J\right)=\mathcal{C}(J) \mathcal{C}\left(Q_{1}\right)+\mathcal{S}\left(Q_{1}\right) \mathcal{S}(J) .
\end{aligned}
$$

We have

$$
\mathcal{X}(G)=\left(V \mathcal{C}\left(Q_{1}\right)+W \mathcal{S}\left(Q_{1}\right)\right) \mathcal{S}(J)+\left(V \mathcal{S}\left(Q_{1}\right)+W \mathcal{C}\left(Q_{1}\right)\right) \mathcal{C}(J) .
$$

With (5.8) and (4.3), this implies

$$
\begin{aligned}
V_{1} \mathcal{L}(J)= & -\left(W_{3}+P V_{1}\right) \mathcal{C}(J)+\frac{1}{2} W_{1} F-\frac{1}{2}\left(V \mathcal{C}_{2}(Q)+W \mathcal{S}_{2}(Q)\right) F^{2} \\
& +\frac{1}{2} W_{2} \mathcal{C}_{2}(F)+\mu(\varepsilon, u) \\
V_{1} & =V \mathcal{C}\left(Q_{1}\right)+W \mathcal{S}\left(Q_{1}\right) \\
W_{3} & =V \mathcal{S}\left(Q_{1}\right)+W \mathcal{C}\left(Q_{1}\right) \\
P & =\mathcal{S}\left(\left(1-u^{2}\right) d^{2}\right) / \mathcal{C}\left(\left(1-u^{2}\right) d^{2}\right)=-u d+\mathcal{O}\left(d^{2}\right) \\
\mathcal{L}(J) & =\mathcal{S}(J)-P \cdot \mathcal{C}(J) .
\end{aligned}
$$


We divide (5.12) by $\left(1-u^{2}\right) d^{2}$, this implies

$$
\begin{aligned}
\frac{V_{1}}{\left(1-u^{2}\right) d^{2}} \mathcal{L}(J)= & -\frac{W_{3}+P V_{1}}{\left(1-u^{2}\right) d^{2}} \mathcal{C}(J)+\frac{W_{1}}{2\left(1-u^{2}\right) d^{2}} F-\frac{V \mathcal{C}_{2}(Q)+W \mathcal{S}_{2}(Q)}{2\left(1-u^{2}\right) d^{2}} F^{2} \\
& +\frac{W_{2}}{2\left(1-u^{2}\right) d^{2}} \mathcal{C}_{2}(F)+\frac{\mu(\varepsilon, u)}{\left(1-u^{2}\right) d^{2}}
\end{aligned}
$$

where $F$ and $J$ are coupled by the equation $Q_{1} J=\mathcal{C}(Q F)=\mathcal{C}(G)$.

Theorem 5.1. With the above notations, we have the following estimation

$$
\|d D \mathcal{C}(G)\|_{n+1}=\mathcal{O}\left((n-7) ! \pi^{-n}\right) \quad \text { as } n \rightarrow \infty \text {. }
$$

Proof. We set

$$
e_{n}:=\frac{\|d D \mathcal{C}(G)\|_{n+1}}{(n-6) ! \pi^{-n}} \text { for } n \geq 7
$$

We must show that $e_{n}=\mathcal{O}\left(n^{-1}\right)$. In the sequel we will use the convention : If $a_{n}, n=0,1, \ldots$ is any sequence of positive real numbers, then

$$
a_{n}^{+}:=\max \left(a_{0}, a_{1}, \ldots a_{n}\right), \text { for all } n \geq 0 .
$$

In this proof $K_{1}, K_{2}, \ldots$ will always denote constants independent of $n$. Theorem 4.3 gives

$$
\begin{gathered}
\|G\|_{n} \leq K_{1} e_{n}^{+}(n-1) ! \pi^{-n} \\
\left\|G^{2}\right\|_{n} \leq K_{2} f_{n}(n-10) ! \pi^{-n}, \quad \text { for } n \geq 18,
\end{gathered}
$$

where

$$
f_{n}=\sum_{i=9}^{n-9} e_{i}^{+} e_{n-i}^{+} \frac{(i-1) !(n-i-1) !}{(n-10) !}, \quad \text { for } n \geq 18 .
$$

Using theorem 3.3, (5), we find

$$
\begin{aligned}
& \|F\|_{n} \leq K_{4} e_{n+1}^{+}(n+1) ! \pi^{-n}, \\
& \left\|F^{2}\right\|_{n} \leq K_{3} f_{n+4}(n-6) ! \pi^{-n} .
\end{aligned}
$$

Using theorem 4.2 , we obtain

$$
\left\|\mathcal{C}_{2}(F)\right\|_{n} \leq K_{5} e_{n+1}^{+}(n+1) ! \pi^{-n} .
$$

Observe that the convergent polynomials series $W_{1} / 2\left(1-u^{2}\right) d^{2}, W_{2} / 2\left(1-u^{2}\right) d^{2}$ begins with $d^{9}$ and $\left(V \mathcal{C}_{2}(Q)+W \mathcal{S}_{2}(Q)\right) / 2\left(1-u^{2}\right) d^{2}$ begin with 1 . Using theorem 4.5 we thus obtain

$$
\left\|\frac{W_{2}}{2\left(1-u^{2}\right) d^{2}} \mathcal{C}_{2}(F)\right\|_{n} \leq K_{6} e_{n-7}^{+}(n-7) ! \pi^{-n}
$$




$$
\begin{gathered}
\left\|\frac{W_{1}}{2\left(1-u^{2}\right) d^{2}} F\right\|_{n} \leq K_{7} e_{n-7}^{+}(n-7) ! \pi^{-n}, \\
\left\|\frac{\left(V \mathcal{C}_{2}(Q)+W \mathcal{S}_{2}(Q)\right)}{2\left(1-u^{2}\right) d^{2}} F^{2}\right\|_{n} \leq K_{8} f_{n+4}^{+}(n-6) ! \pi^{-n} .
\end{gathered}
$$

On the other hand

$$
\left\|Q_{1} J\right\|_{n}=\|\mathcal{C}(G)\|_{n} \leq e_{n}(n-6) ! \pi^{-n} .
$$

We apply the theorems 3.3, (5) and 4.2 and find

$$
\|\mathcal{C}(J)\|_{n} \leq K_{9} e_{n+2}(n-4) ! \pi^{-n} .
$$

Because the convergent polynomial series $\left(W_{3}+P V_{1}\right) /\left(1-u^{2}\right) d^{2}=\mathcal{O}\left(d^{3}\right)$, then

$$
\left\|\frac{\left(W_{3}+P V_{1}\right)}{\left(1-u^{2}\right) d^{2}} \mathcal{C}(J)\right\|_{n} \leq K_{10} e_{n-1}^{+}(n-9) ! \pi^{-n} .
$$

With a crude estimate of the convergent terms $\mu(\varepsilon, u)$ the inequalities (5.21)(5.24) gives

$$
\left\|\frac{V_{1}}{2\left(1-u^{2}\right) d^{2}} \mathcal{L}(J)\right\|_{n} \leq K_{11}\left(1+e_{n-1}^{+}+f_{n+4}^{+}\right)(n-6) ! \pi^{-n} .
$$

The multiplication by the convergent terme $2\left(1-u^{2}\right) d^{2} / V_{1}$ only changes the constant $K_{11}$. Since theorem 4.5 applies to the operator $\mathcal{L}$ defined in (4.3), we obtain

$$
\|d D J\|_{n} \leq K_{12}\left(1+e_{n-1}^{+}+f_{n+4}^{+}\right)(n-6) ! \pi^{-n} .
$$

This with theorem 4.5 gives via

$$
d D \mathcal{C}(G)=d D\left(Q_{1} J\right)=J \cdot\left(d D Q_{1}\right)+Q_{1} \cdot(d D J),
$$

the estimate

$$
\|d D \mathcal{C}(G)\|_{n} \leq K_{13}\left(1+e_{n-3}^{+}+f_{n+2}^{+}\right)(n-8) ! \pi^{-n} .
$$

By equation (5.15), we obtain

$$
e_{n-1} \leq \frac{K}{n}\left(1+e_{n-3}^{+}+f_{n+2}^{+}\right) .
$$

Lemma 5.2. Under the condition (5.2\%), we have $e_{n}=\mathcal{O}\left(n^{-1}\right)$ as $n \rightarrow \infty$.

Proof. Let $K_{1} \geq 9 ! e_{10}^{+}$an arbitrary number. We assume that

$$
e_{n} \leq \frac{K_{1}(n+p) !}{(n-1) !(p+10) !}, \quad \text { for } 10 \leq n \leq N-3,
$$


with $p \geq-1, N \geq 14$. This gives for $13 \leq n \leq N$

$$
(n-8) ! f_{n+2} \leq 2 e_{9}^{+} K_{1} \frac{8 !(n+p-7) !}{!(p+10) !}+K_{1}^{2} \sum_{i=10}^{n-8} \frac{(i+p) !(n+p-i+2) !}{((p+10) !)^{2}} .
$$

Using the inequality,

$$
\sum_{i=10}^{n-8}(i+p) !(n+p-i+2) ! \leq(p+10) !(n+p-7) !
$$

we obtain

$$
f_{n+2} \leq K_{2} \frac{(n+p-7) !}{(p+10) !(n-8) !} \leq K_{2} \frac{(n+p-3) !}{(p+10) !(n-4) !} \quad \text { for } 13 \leq n \leq N,
$$

with a constant $K_{2}$ depends on $k_{1}$, independent of $p$. The asumption (5.27) of the lemma yields

$$
e_{n-1} \leq \frac{K}{n}\left(1+K_{1}+K_{2}\right) \frac{(n+p-3) !}{(p+10) !(n-4) !} \quad \text { for } 13 \leq n \leq N,
$$

this implies

$$
e_{n} \leq \frac{K_{3}}{n+1} \frac{(n+p) !}{(p+10) !(n-1) !}, \quad \text { for } 12 \leq n \leq N-1,
$$

with a constant $K_{3}$ only depending upon $K_{1}$. Now we choose $N_{0} \geq 12$ so large that $\frac{K_{3}}{N_{0}} \leq K_{1}$ and then $p$ so large that (5.28) holds. Then

$$
e_{n} \leq \frac{K_{1}}{n+1} \frac{(n+p) !}{(p+10) !(n-1) !} \quad \text { for } 12 \leq n \leq N-1 \text {, with } N \geq N_{0} .
$$

Since $K_{1} \geq 9$ ! $e_{10}$ is an arbitrary number, we have shown for any $p \geq-1$, taht

$$
e_{n}=\mathcal{O}\left(\frac{(n+p) !}{(n-1) !}\right) \text { as } n \rightarrow \infty
$$

implies that

$$
e_{n}=\mathcal{O}\left(\frac{(n+p-1) !}{(n-1) !}\right) \text { as } n \rightarrow \infty .
$$

Consequently

$$
e_{n}=\mathcal{O}\left(n^{-1}\right), \text { as } n \rightarrow \infty
$$

Thus we have shown that

$$
\|d D \mathcal{C}(G)\|_{n+1}=\mathcal{O}\left((n-7) ! \pi^{-n}\right) \text { as } n \rightarrow \infty .
$$


Let $E:=\mathcal{C}(G)$. Like $G$, the polynomial series $E$ is odd in $d$ and the coefficients are odd in $u$. We partition them

$$
\begin{aligned}
E_{n}= & \alpha_{n}(n-1) !\left(\frac{i}{\pi}\right)^{n-1} \tau_{n}(u)+\beta_{n-2}(n-3) !\left(\frac{i}{\pi}\right)^{n-3} \tau_{n-2}(u) \\
& +\gamma_{n-4}(n-5) !\left(\frac{i}{\pi}\right)^{n-5} \tau_{n-4}(u)+\bar{E}_{n-6},
\end{aligned}
$$

for odd $n \geq 7$, where $\alpha_{n}$ and $\beta_{n}$ are real number and also $\bar{E}$ have at most degree $n$ for all $n$. For the whole series $E$ this is equivalent to

$$
\mathcal{C}(G)=E=E_{1}+d^{2} E_{2}+d^{4} E_{4}+d^{6} \bar{E},
$$

where

$$
\begin{aligned}
E_{1} & =\sum_{n=7}^{+\infty} \alpha_{n}(n-1) !\left(\frac{i}{\pi}\right)^{n-1} \tau_{n}(u) d^{n}, \\
E_{2} & =\sum_{n=5}^{+\infty} \beta_{n}(n-1) !\left(\frac{i}{\pi}\right)^{n-1} \tau_{n}(u) d^{n}, \\
E_{3} & =\sum_{n=3}^{+\infty} \gamma_{n}(n-1) !\left(\frac{i}{\pi}\right)^{n-1} \tau_{n}(u) d^{n}, \\
\bar{E} & =\sum_{n=1}^{+\infty} \bar{E}_{n}(u) d^{n} .
\end{aligned}
$$

Theorem 5.1 and the definition of the norms yields

$\alpha_{n}=\mathcal{O}\left(n^{-7}\right), \beta_{n}=\mathcal{O}\left(n^{-5}\right), \gamma_{n}=\mathcal{O}\left(n^{-3}\right)$ and $\left\|D \bar{E}_{n}\right\|_{n+1}=\mathcal{O}\left((n-1) ! \pi^{-n}\right)$.

We apply $\mathcal{C}^{-1}$ to $(5.29)$ and obtain

$$
G=\mathcal{C}^{-1}\left(E_{1}\right)+d^{2} \mathcal{C}^{-1}\left(E_{2}\right)+d^{4} \mathcal{C}^{-1}\left(E_{4}\right)+d^{6} \mathcal{C}^{-1}(\bar{E}) .
$$

To the first two summands, theorem 4.4 applies and yields

$$
\begin{aligned}
\left\|\left\{\mathcal{C}^{-1}\left(E_{1}\right)\right\}_{n}-\alpha(n-1) !\left(\frac{i}{\pi}\right)^{n-1} \tau_{n}(u)\right\|_{n} & =\mathcal{O}\left((n-7) ! \pi^{-n}\right), \\
\left\|\left\{\mathcal{C}^{-1}\left(E_{2}\right)\right\}_{n}-\beta(n-1) !\left(\frac{i}{\pi}\right)^{n-1} \tau_{n}(u)\right\|_{n} & =\mathcal{O}\left((n-5) ! \pi^{-n}\right), \\
\left\|\left\{\mathcal{C}^{-1}\left(E_{3}\right)\right\}_{n}-\gamma(n-1) !\left(\frac{i}{\pi}\right)^{n-1} \tau_{n}(u)\right\|_{n} & =\mathcal{O}\left((n-3) ! \pi^{-n}\right),
\end{aligned}
$$

where

$$
\alpha=\frac{1}{\pi} \sum_{n=7}^{\infty} \alpha_{n}, \beta=\frac{1}{\pi} \sum_{n=5}^{\infty} \beta_{n}, \gamma=\frac{1}{\pi} \sum_{n=3}^{\infty} \gamma_{n} .
$$


To the last part of (5.29), we apply theorem 4.3 and obtain

$$
\left\|\left\{\mathcal{C}^{-1}(\bar{E})\right\}_{n}\right\|_{n}=\mathcal{O}\left((n-1) ! \pi^{-n} \log (n)\right) .
$$

Using $G=\mathcal{C}^{-1}(E)$, we have shown

$$
\begin{gathered}
\left\|G_{n}-\alpha(n-1) !\left(\frac{i}{\pi}\right)^{n-1} \tau_{n}-\beta(n-3) !\left(\frac{i}{\pi}\right)^{n-3} \tau_{n-2}-\gamma(n-5) !\left(\frac{i}{\pi}\right)^{n-5} \tau_{n-4}\right\|_{n} \\
=\mathcal{O}\left((n-7) ! \pi^{-n} \log (n)\right) .
\end{gathered}
$$

If we use (5.3) and the relation $\tau_{n+1}=\frac{1}{n} D \tau_{n}$ and we apply theorem 3.3, (5), we obtain our final result of this section

$$
\begin{gathered}
\left\|F_{n}-\alpha n !\left(\frac{i}{\pi}\right)^{n+1} \tau_{n+1}^{\prime}-\beta(n-2) !\left(\frac{i}{\pi}\right)^{n-1} \tau_{n-1}^{\prime}-\gamma(n-4) !\left(\frac{i}{\pi}\right)^{n-3} \tau_{n-3}^{\prime}\right\|_{n}= \\
\mathcal{O}\left((n-5) ! \pi^{-n} \log (n+2)\right) .
\end{gathered}
$$

and

$$
\begin{aligned}
\| F_{n}-\alpha(n+1) !\left(\frac{i}{\pi}\right)^{n+1} \frac{\tau_{n+2}}{\tau_{2}} & -\beta(n-1) !\left(\frac{i}{\pi}\right)^{n-1} \frac{\tau_{n}}{\tau_{2}}-\gamma(n-3) !\left(\frac{i}{\pi}\right)^{n-3} \frac{\tau_{n-2}}{\tau_{2}} \|_{n} \\
& =\mathcal{O}\left((n-5) ! \pi^{-n} \log (n+2)\right) .
\end{aligned}
$$

\section{Preparation of the functions to construct a quasi- solutions}

In the previous section, we have shown that equation (2.4) has a formal solution and we found an asymptotic approximation of the coefficients of this formal solution. We will use this to construct the quasi-solution. To that purpose, we define the functions

$$
\begin{gathered}
H_{n}(u):=(n+1) !\left(\frac{i}{\pi}\right)^{n+1} \tau_{n+2}(u) \\
h(t, u):=\sum_{\substack{n=7 \\
n \text { odd }}}^{\infty} H_{n}(u) \frac{t^{n-1}}{(n-1) !} .
\end{gathered}
$$

We rewrite

$$
h(t, u)=\left(\frac{i}{\pi}\right)^{2} \sum_{\substack{n=6 \\ n \text { even }}}^{\infty}(n+1)(n+2)\left(\frac{i t}{\pi}\right)^{n} \tau_{n+3}(u) .
$$


Using proposition (3.1)-(4), we obtain

$$
h(t, u)=\frac{-1}{\pi^{2}} \sum_{\substack{n=6 \\ n \text { even }}}^{\infty} \frac{1}{n !}\left(\frac{i t}{\pi}\right)^{n} \frac{d^{n}}{d^{n} \xi}\left(\frac{d^{2}}{d^{2} \xi}(\tanh (\xi))\right),
$$

or equivalently

$$
h(t, u)=\frac{-1}{\pi^{2}} \sum_{\substack{n=6 \\ n \text { even }}}^{\infty} \frac{1}{n !}\left(\frac{i t}{\pi}\right)^{n} \frac{d^{n}}{d^{n} \xi}(g(\xi)),
$$

where $g(\xi)=-2\left(\tanh (\xi)-\tanh (\xi)^{3}\right)$ and $\xi=\xi(u)=\operatorname{artanh}(u)$.

and hence that

$$
h(t, u)=\frac{-1}{\pi^{2}} \sum_{\substack{n=0 \\ n \text { even }}}^{\infty} \frac{1}{n !}\left(\frac{i t}{\pi}\right)^{n} \frac{d^{n}}{d^{n} \xi}(g(\xi))+\mu(t, u),
$$

where

$$
\mu(t, u):=-\frac{2 u}{\pi^{2}} \tau_{2}(u)+\frac{4}{\pi^{4}}\left(3 u^{3}-2 u\right) \tau_{2}(u) t^{2}-\frac{2}{3 \pi^{6}}\left(45 u^{5}-60 u^{3}+17 u\right) \tau_{2}(u) t^{4} .
$$

Thus we can write

$$
h(t, u)=\frac{-1}{2 \pi^{2}}\left[g\left(\xi+\frac{i t}{\pi}\right)+g\left(\xi-\frac{i t}{\pi}\right)\right]+\mu(t, u) .
$$

This gives

$$
\begin{aligned}
h(t, u) & =\left(1-u^{2}\right)\left[\frac{\left(4 u^{3}-4 u\right) \sin \left(\frac{t}{\pi}\right)^{4}-\left(6 u^{3}-2 u\right) \sin \left(\frac{t}{\pi}\right)^{2}+2 u}{\pi^{2}\left(\cos \left(\frac{t}{\pi}\right)^{2}+u^{2} \sin \left(\frac{t}{\pi}\right)^{2}\right)^{3}}\right] \\
& +\mu(t, u) .
\end{aligned}
$$

For fixed real $u$ the function $h(., u)$ is analytic in $|t|<\rho$, where $\rho=\frac{\pi^{2}}{2}$. In the subsequent definition, we consider real values of $u, 0<u \leq 1$, here $h(., u)$ is also analytic with respect to $t$ on the positive real axis.

Now we define the function $\mathcal{H}(d, u)$ by

$$
\mathcal{H}(d, u):=\int_{0}^{+\infty} e^{-\frac{t}{d}} h(t, u) d t, \quad \text { for } \quad(0<u \leq 1) .
$$

The function $\mathcal{H}(d,$.$) is real analytic ; they can be continued analytically to the$ interval $-1<u \leq 1$ in the following way. Choose some positive number $M$ and let $\Gamma_{1}$ the path consisting of the segment from 0 to $M i$ and of the ray $t \mapsto t+M i, t \geq 0$. 
Let $\Gamma_{2}$ the symmetric path that could also be obtained using $-M$ instead of $M$. Recalling (6.4), we can also define

$$
\begin{aligned}
\mathcal{H}(d, u): & =-\frac{1}{\pi^{2}}\left[\int_{\Gamma_{2}} e^{-\frac{t}{d}} g\left(\xi+\frac{i t}{\pi}\right) d t+\int_{\Gamma_{1}} e^{-\frac{t}{d}} g\left(\xi-\frac{i t}{\pi}\right) d t\right] \\
& +\mu_{1}(d, u)
\end{aligned}
$$

where

$$
\mu_{1}(d, u):=\int_{0}^{\infty} e^{-\frac{t}{d}} \mu(t, u) d t,
$$

for $-\tanh \left(\frac{2}{\pi} M\right)<u \leq 1$, where $\xi=\operatorname{artanh}(u)$, because the singularities of tanh are $i\left(\frac{\pi}{2}+n \pi\right), n$ integer. As $M$ is arbitrary, this defines the analytic continuation of $\mathcal{H}(d,$.$) for -1<u \leq 1$.

In the sequel we consider $\left.\left.u_{0} \in\right]-1,0\right]$.

Lemma 6.1. If we consider the above function $\mathcal{H}(d, u)$ and the operators defined in (2.29). Then, for $u_{0}<u \leq 1$

-1 .

$$
\mathcal{S}_{2}(\mathcal{H})=\left(1-u^{2}\right) \mathcal{D}_{1}(d, u)
$$

where the function $\mathcal{D}_{1}(d, u)$ is analytic, beginnings with $d^{8}$.

$-2$.

$$
\mathcal{C}_{2}(\mathcal{H})=-\mathcal{H}(d, u)+\left(1-u^{2}\right) \mathcal{D}_{2}(d, u),
$$

where the function $\mathcal{D}_{2}(d, u)$ is analytic, beginnings with $d^{7}$. $-3$

$$
\mathcal{S}_{2}\left(\frac{1}{\tau_{2}(u)} \mathcal{H}\right)=-\frac{2 u \varepsilon}{\tau_{2}(u)} \mathcal{H}(d, u)+\mathcal{D}_{3}(d, u)
$$

where the function $\mathcal{D}_{3}(d, u)$ is analytic beginnings with $d^{8}$

Proof. (1)- If we replace $u$ by $T^{+}$and $T^{-}$in (6.5) we obtain

$$
\mathcal{H}\left(d, T^{+}\right)-\mathcal{H}\left(d, T^{-}\right)=\int_{0}^{+\infty} e^{-\frac{t}{d}}\left(h\left(t, T^{+}\right)-h\left(t, T^{-}\right)\right) d t
$$

this implies

$$
\mathcal{H}\left(d, T^{+}\right)-\mathcal{H}\left(d, T^{-}\right)=-\frac{1}{2 \pi^{2}}\left(\mathcal{I}^{+}-\mathcal{I}^{-}\right)+\sigma\left(d, T^{+}\right)-\sigma\left(d, T^{-}\right),
$$

where

$$
\begin{aligned}
\mathcal{I}^{+} & =\int_{0}^{+\infty} e^{-\frac{t}{d}}\left(g\left(\xi\left(T^{+}\right)+\frac{i t}{\pi}\right)+g\left(\xi\left(T^{+}\right)-\frac{i t}{\pi}\right)\right) d t \\
\mathcal{I}^{-} & =\int_{0}^{+\infty} e^{-\frac{t}{d}}\left(g\left(\xi\left(T^{-}\right)+\frac{i t}{\pi}\right)+g\left(\xi\left(T^{-}\right)-\frac{i t}{\pi}\right)\right) d t \\
\sigma(d, u) & =\int_{0}^{+\infty} e^{-\frac{t}{d}} \mu(t, u) d t .
\end{aligned}
$$


Using $\xi\left(T^{ \pm}\right)=\xi \pm d$, where $\xi$ and $u$ are coupled by $\xi=\operatorname{artanh}(u)$, we obtain

$$
\mathcal{I}^{+}=\int_{0}^{+\infty} e^{-\frac{t}{d}} g\left(\xi+d+\frac{i t}{\pi}\right) d t+\int_{0}^{+\infty} e^{-\frac{t}{d}} g\left(\xi+d-\frac{i t}{\pi}\right) d t .
$$

If we substitute $t+\pi i d$ in the first part, $t-\pi i d$ in the second part, we obtain

$$
\mathcal{I}^{+}=-\int_{-\pi i d}^{+\infty+\pi i d} e^{-\frac{t}{d}} g\left(\xi+\frac{i t}{\pi}\right) d t-\int_{\pi i d}^{+\infty+\pi i d} e^{-\frac{t}{d}} g\left(\xi-\frac{i t}{\pi}\right) d t .
$$

We apply Cauchy's theorem

$$
\begin{aligned}
\mathcal{I}^{+}=-\int_{0}^{+\infty} e^{-\frac{t}{d}}\left(g\left(\xi+\frac{i t}{\pi}\right)+g\left(\xi-\frac{i t}{\pi}\right)\right) d t & +\int_{0}^{-\pi i d} e^{-\frac{t}{d}} g\left(\xi+\frac{i t}{\pi}\right) d t \\
& +\int_{0}^{\pi i d} e^{-\frac{t}{d}} g\left(\xi-\frac{i t}{\pi}\right) d t .
\end{aligned}
$$

Substitute $t=-i s d$ in the second part, $t=i s d$ in the third part, we obtain

$$
\mathcal{I}^{+}=-\int_{0}^{+\infty} e^{-\frac{t}{d}}\left(g\left(\xi+\frac{i t}{\pi}\right)+g\left(\xi-\frac{i t}{\pi}\right)\right) d t+2 d \int_{0}^{\pi} \sin (s) g\left(\xi+\frac{s d}{\pi}\right) d s .
$$

This implies

$$
\mathcal{I}^{+}=-\int_{0}^{+\infty} e^{-\frac{t}{d}}\left(g\left(\xi+\frac{i t}{\pi}\right)+g\left(\xi-\frac{i t}{\pi}\right)\right) d t-4 d\left(1-u^{2}\right) \mathcal{D}^{+}(d, u),
$$

where

$$
\mathcal{D}^{+}(d, u)=\int_{0}^{\pi} \sin (s) \frac{\left(u+\tanh \left(\frac{s d}{\pi}\right)\right)\left(1-\tanh \left(\frac{s d}{\pi}\right)^{2}\right)}{\left(1+u \tanh \left(\frac{d s}{\pi}\right)\right)^{3}} d s
$$

We can also use the same method for $\mathcal{I}^{-}$and obtain

$$
\mathcal{I}^{-}=-\int_{0}^{+\infty} e^{-\frac{t}{d}}\left(g\left(\xi+\frac{i t}{\pi}\right)+g\left(\xi-\frac{i t}{\pi}\right)\right) d t-4 d\left(1-u^{2}\right) \mathcal{D}^{-}(d, u),
$$

where

$$
\mathcal{D}^{-}(d, u)=\int_{0}^{\pi} \sin (s) \frac{\left(u-\tanh \left(\frac{d s}{\pi}\right)\right)\left(1-\tanh \left(\frac{s d}{\pi}\right)^{2}\right)}{\left(1-u \tanh \left(\frac{s d}{\pi}\right)\right)^{3}} d s .
$$

Consequently

$\mathcal{H}\left(d, T^{+}\right)-\mathcal{H}\left(d, T^{-}\right)=\frac{2 d\left(1-u^{2}\right)}{\pi^{2}}\left(\mathcal{D}^{+}(d, u)-\mathcal{D}^{-}(d, u)\right)+\sigma\left(d, T^{+}\right)-\sigma\left(d, T^{-}\right)$.

Using (2.22), we find

$$
\mathcal{S}_{2}(\mathcal{H})=\left(1-u^{2}\right) \mathcal{D}_{1}(d, u)
$$


where $\mathcal{D}_{1}(d, u)$ is analytic, beginnings with $d^{8}$.

(2). Using the same method, we obtain

$$
\mathcal{H}\left(d, T^{+}\right)+\mathcal{H}\left(d, T^{-}\right)=-2 \mathcal{H}(d, u)+2\left(1-u^{2}\right) \mathcal{D}_{2}(d, u) .
$$

With (2.22) we find

$$
\mathcal{C}_{2}(\mathcal{H})=-\mathcal{H}(d, u)+\left(1-u^{2}\right) \mathcal{D}_{2}(d, u) .
$$

$\mathcal{D}_{2}(d, u)$ is analytic, beginnings with $d^{7}$.

(3). Using (5.7), (2.8), (2.22) and (1), (2) of this lemma, we obtain

$$
\begin{aligned}
\mathcal{S}_{2}\left(\frac{1}{\tau_{2}(u)} \mathcal{H}\right) & =\mathcal{S}_{2}\left(\frac{1}{\tau_{2}(u)}\right) \mathcal{C}_{2}(\mathcal{H})+\mathcal{C}_{2}\left(\frac{1}{\tau_{2}(u)}\right) \mathcal{S}_{2}(\mathcal{H}) \\
& =-\frac{2 u \varepsilon}{\tau_{2}(u)} \mathcal{H}(d, u)+\mathcal{D}_{3}(d, u)
\end{aligned}
$$

where

$$
\mathcal{D}_{3}(d, u):=\left(1+\left(1+u^{2}\right) \sinh (d)^{2}\right)\left(1-u^{2}\right) \mathcal{D}_{1}(d, u)+2 u \varepsilon\left(1-u^{2}\right) \mathcal{D}_{2}(d, u) .
$$

Proposition 6.2. We have

1. For $u_{0}<u \leq 1$

$$
\mathcal{H}(d, u) \sim \sum_{\substack{n=7 \\ n \text { odd }}}^{\infty}(n+1) !\left(\frac{i}{\pi}\right)^{n+1} \tau_{n+2}(u) d^{n} \text {, as } d \searrow 0,
$$

2. $\left|\frac{\partial \mathcal{H}}{\partial u}(d, u)\right| \leq K d \quad$ for $u_{0} \leq u \leq 1 \quad(d>0)$.

\section{Proof.}

1. To prove (1) we use Watson's lemma and (6.2). if $\left|t-k \pi^{2}\right|<\frac{\pi^{2}}{2}$, for $u_{0} \leq u<1$

2. - For $u_{0} \leq u \leq \frac{1}{2}$

$$
|\mathcal{H}(d, u)|=\frac{1}{d^{6}} \int_{0}^{\infty} e^{-t / d} h^{(-6)}(t, u) d t
$$

where $h^{(-6)}(t, u)$ satisfies

$$
\left(\frac{\partial}{\partial t}\right)^{6} h^{(-6)}=\frac{\partial h}{\partial u} \text { and }\left(\frac{\partial}{\partial t}\right)^{k} h^{(-6)}(0, u)=0, \quad \text { for } k=0, \ldots, 5 .
$$

Because of

$$
h^{(-6)}(t, u) \leq K \frac{t^{7}}{7 !} \quad \text { for } t \geq 0
$$


we obtain

$$
\left|\frac{\partial \mathcal{H}}{\partial u}(d, u)\right| \leq \frac{K}{7 ! d^{6}} \int_{0}^{\infty} e^{-\frac{t}{d}} t^{7} d t
$$

This implies

$$
\left|\frac{\partial \mathcal{H}}{\partial u}(d, u)\right| \leq K d \quad(d>0) .
$$

- for $\frac{1}{2} \leq u \leq 1$

$$
\begin{aligned}
\left|\frac{\partial \mathcal{H}}{\partial u}(d, u)\right| & =\left|\int_{0}^{\infty} e^{-\frac{t}{d}} \frac{\partial h}{\partial u}(d, u) d t\right| \\
& \leq K \int_{0}^{\infty} e^{-\frac{t}{d}} d t \\
& \leq K d \quad(d>0) .
\end{aligned}
$$

Let us consider a sequence $R_{n}(u)$ of polynomials of degree at most $n$, such that

$$
\left\|R_{n}\right\|_{n}=O\left((n-5) ! \pi^{-1} \log (n)\right)
$$

Lemma 6.3. Let polynomial series difine $R \in \mathcal{Q}$ with the above estimate be given and define

$$
\begin{aligned}
r(t, u): & =\sum_{n=7}^{\infty} R_{n}(u) \frac{t^{n-1}}{(n-1) !}, \quad\left(t \in \mathbb{C},|t| \leq \frac{\pi^{2}}{2}, u_{0} \leq u \leq 1\right), \\
r(t, u): & =r\left(\frac{\pi^{2}}{2}, u\right)+\left(t-\frac{\pi^{2}}{2}\right) \frac{\partial r}{\partial t}\left(\frac{\pi^{2}}{2}, u\right), \quad\left(t>\frac{\pi^{2}}{2}, u_{0} \leq u \leq 1\right), \\
\mathcal{R}(d, u): & =\int_{0}^{\infty} e^{-\frac{t}{d}} r(t, u) d t .
\end{aligned}
$$

Then

1. $r$ is continuously differentiable function on the set $B$ of all $(t, u)$ such that $u$ satisfies $u_{0} \leq u \leq 1$ and $t$ is a complex number and satisfies $|t| \leq \frac{\pi^{2}}{2}$ or $t>\frac{\pi^{2}}{2}$. The restriction of $r$ to $u_{0} \leq u \leq 1,|t| \leq \frac{\pi^{2}}{2}$ is twice continuously differentiable. For fixed $u_{0} \leq u \leq 1$ the function $r(t, u)$ is analytic in $|t|<\frac{\pi^{2}}{2}$.

2. $\mathcal{R}(d, u)$ is continuous, partially differentiable with respect to $u$, has continuous partial derivative and

$$
\mathcal{R}(d, u) \sim \sum_{n=7}^{\infty} R_{n}(u) d^{n}, \text { as } d \searrow 0
$$

3. $|\mathcal{R}(d, u)| \leq K d^{3},\left|\frac{\partial \mathcal{R}}{\partial u}(d, u)\right| \leq K d^{3}$, for $u_{0} \leq u \leq 1 \quad(d>0)$. 
The importance of our definition of $\mathcal{R}$ lies in a certain compatibility with insertion of the functions $T^{+}, T^{-}$for $u$. First let

$$
\begin{aligned}
\sum_{n=7}^{\infty} R_{n}^{+}(u) d^{n} & :=\sum_{n=7}^{\infty} R_{n}\left(T^{+}(d, u)\right) d^{n}, \\
\sum_{n=7}^{\infty} R_{n}^{-}(u) d^{n} & :=\sum_{n=7}^{\infty} R_{n}\left(T^{-}(d, u)\right) d^{n} .
\end{aligned}
$$

We obtain a new sequences $R_{n}^{+}(u), R_{n}^{-}(u)$ of polynomials of degree at most $n$. Theorem 4.2, and (4.2) imply

$$
\begin{aligned}
\left\|R_{n}^{+}(u)\right\|_{n} & =O\left((n-5) ! \pi^{-n} \log (n)\right) \\
\left\|R_{n}^{-}(u)\right\|_{n} & =O\left((n-5) ! \pi^{-n} \log (n)\right)
\end{aligned}
$$

Therefore we can use lemma 6.3 for $R_{n}^{+}(u), R_{n}^{-}(u)$, and obtain functions $\mathcal{R}^{+}(d, u)$, $\mathcal{R}^{-}(d, u)$.

Theorem 6.4. There is a positive constant $K$ independent of $d, u$ such that

$$
\begin{aligned}
& \left|\mathcal{R}^{+}(d, u)-\mathcal{R}\left(d, T^{+}\right)\right| \leq K d^{3} e^{-\frac{\pi^{2}}{2 d}} \quad \text { for } \quad\left(0<d<d_{0}, u_{0}<u<1\right) \\
& \left|\mathcal{R}^{-}(d, u)-\mathcal{R}\left(d, T^{-}\right)\right| \leq K d^{3} e^{-\frac{\pi^{2}}{2 d}} \quad \text { for } \quad\left(0<d<d_{0}, u_{0}<u<1\right) .
\end{aligned}
$$

Proof The proof is exactly the one of [5].

Definition 6.5. Let $\mathcal{D}(d, u)$ be a function defined for $0<d<d_{0}$ and $u_{0}<u<1$. We say that $\mathcal{D}(d, u)$ has property $G$ if

$$
\mathcal{D}(d, u)=\int_{0}^{\infty} e^{-\frac{t}{d}} q(t, u) d t \quad\left(0<d<d_{0}, u_{0}<u<1\right)
$$

is the Laplace transform of some function $q(t, u)$ that has the following proprties :

1. $q(t, u)$ is defined if $u_{0}<u<1$ and either $t$ is complex and $|t|<\frac{\pi^{2}}{2}$ or $t$ is real and $t \geq 0$,

2. $q(t, u)$ is analytic in $|t|<\frac{\pi^{2}}{2}$ for $u_{0}<u<1$,

3. $q(t, u)$ restricted to $0 \leq t<\frac{\pi^{2}}{2}$ or $t \geq \frac{\pi^{2}}{2}$ is continuous and the $\lim _{t \rightarrow \frac{\pi^{2}}{2}} q(t, u)$ exists for every $u_{0}<u<1$,

4. there is a positive constant $K$ such that

$$
|q(t, u)| \leq K e^{K t}, \text { for } t \geq 0, u_{0}<u<1,\left(0<d<d_{0}, u_{0}<u<1\right)
$$

The set of all function with the property $G$ will be denote by $\mathcal{G}$

Lemma 6.6. For $u_{0}<u \leq 1$ 
1. If $\mathcal{H}(d, u)$ is the function defined in (6.6) then

$$
d^{4} \mathcal{H}(d, u)=\left(1-u^{2}\right) \tilde{\mathcal{H}}(d, u)+\mathcal{O}\left(\left(1-u^{2}\right) e^{-\frac{\pi^{2}}{2 d}}\right)
$$

where $\tilde{\mathcal{H}}(d, u)$ has property $G$

2. Let $k$ be a psitive integer. If $\mathcal{D}_{1}, \mathcal{D}_{2}$ have property $G$ and their first terms in the Taylor development at $d=0$, begin with $d^{k}$ then

$$
\mathcal{D}_{1}(d, u) \mathcal{D}_{2}(d, u)=d^{k} \mathcal{D}(d, u)+\mathcal{O}\left(d^{k} e^{-\frac{\pi^{2}}{2 d}}\right),
$$

where $\mathcal{D}(d, u)$ has property $G$

3. If $\mathcal{H}(d, u)$ is the function defined in (6.0) then

$$
\mathcal{H}(d, u)^{2}=\left(1-u^{2}\right)^{2} d^{3} \mathcal{E}(d, u)+\mathcal{O}\left(\left(1-u^{2}\right)^{2} d^{3} e^{-\frac{\pi^{2}}{2 d}}\right)
$$

where $\mathcal{E}(d, u)$ has property $G$

4. Any function $\mathcal{D}(d, u)$ analytic in a neighborhood of $d=0$ has property $G$ if $\mathcal{D}(0, u)=0$ for all $u$,

5. If $\mathcal{R}(d, u)$ is defined by lemma 6.3 then $\frac{1}{d^{2}} \mathcal{R}(d, u)$ has property $G$

6. If $\mathcal{D}_{1}, \mathcal{D}_{2}$ have property $G$ then so do $\mathcal{D}_{1}+\mathcal{D}_{2}, \mathcal{D}_{1}-\mathcal{D}_{2}$ and $\mathcal{D}_{1} \cdot \mathcal{D}_{2}$

7. If $\mathcal{D}(d, u)$ has property $G$ then

$$
|\mathcal{D}(d, u)| \leq K d \quad\left(0<d<\frac{1}{K}\right)
$$

with some constant $K>0$ independent of $u$.

\section{Proof.}

1. For $u>0$, we have

$$
d^{4} \mathcal{H}=\left(1-u^{2}\right) \int_{0}^{\infty} e^{-\frac{t}{d}} g_{4}(t, u) d t
$$

where

$$
g_{4}(t, u)=\frac{1}{\left(1-u^{2}\right)} \int_{0}^{t} \int_{0}^{\theta} \int_{0}^{v} \int_{0}^{\tau} h(s, u) d s d \tau d v d \theta
$$

$g_{4}(t, u)$ has a logarithmic singularity at $t_{k}(s)=(2 k+1) \frac{\pi^{2}}{2} \pm d \frac{\pi s}{\varepsilon} i$ for $(k \geq$ $0, s>0)$. it is analytic in $|t|<\frac{\pi^{2}}{2}$ and $\lim _{t \rightarrow \frac{\pi^{2}}{2}} g_{4}(t, u)$ exists.

If we put

$$
\tilde{\mathcal{H}}(d, u)=\int_{0}^{\infty} e^{-\frac{t}{d}} \tilde{g}_{4}(t, u) d t
$$


where

$$
\tilde{g}_{4}(t, u)= \begin{cases}g_{4}(t, u), & \text { if } t \leq \frac{\pi^{2}}{2} \\ g_{4}\left(\frac{\pi^{2}}{2}, u\right), & \text { if } t \geq \frac{\pi^{2}}{2}\end{cases}
$$

then $\tilde{\mathcal{H}}(d, u)$ has property $\mathrm{G}$ and

$$
d^{4} \mathcal{H}(d, u)=\left(1-u^{2}\right) \tilde{\mathcal{H}}(d, u)+\mathcal{O}\left(\left(1-u^{2}\right) e^{-\frac{\pi^{2}}{2 d}}\right) .
$$

(ii)- For $-u_{0}<u<1$, where $0<u_{0}<1$, we have

$$
\begin{aligned}
\mathcal{H}(d, u) & =\int_{0}^{\infty e^{i \varphi}} e^{-\frac{t}{d}} h(t, u) d t+2 \pi i \sum_{k \geq 0} \operatorname{Res}\left(e^{-\frac{t}{d}} h(t, u), t_{k}(s)\right) \\
& =\int_{0}^{\infty e^{i \varphi}} e^{-\frac{t}{d}} h(t, u) d t+\mathcal{O}\left(\frac{1}{d^{2}}\left(1-u^{2}\right) e^{-\frac{\pi^{2}}{2 d}}\right)
\end{aligned}
$$

where $\frac{\pi}{2}<\varphi<\frac{\pi}{4}$. For $0<u<u_{0}$, this formula coincides with the formula

$$
\int_{0}^{\infty} e^{-\frac{t}{d}} h(t, u) d t
$$

and extends it by real analytic continuation for $-u_{0}<u<0$.

This implis

$$
d^{4} \mathcal{H}(d, u)=\left(1-u^{2}\right) \int_{0}^{\infty e^{i \varphi}} e^{-\frac{t}{d}} g_{4}(t, u) d t+\mathcal{O}\left(\left(1-u^{2}\right) d^{2} e^{-\frac{\pi^{2}}{2 d}}\right)
$$

we obtain

$$
\begin{aligned}
d^{4} \mathcal{H}(d, u) & -\left(1-u^{2}\right) \tilde{\mathcal{H}}(d, u)=\left(1-u^{2}\right) \int_{\Gamma} e^{-\frac{t}{d}} g_{2}(t, u) d \Gamma \\
& +\mathcal{O}\left(\left(1-u^{2}\right) e^{-\frac{\pi^{2}}{2 d}}\right)
\end{aligned}
$$

where $\Gamma$ is the path following the real line from infinity to $\left(\frac{\pi^{2}}{2}, 0\right)$, then along the vertical line from $\left(\frac{\pi^{2}}{2}, 0\right)$ to $\left(\frac{\pi^{2}}{2}, \frac{\pi^{2}}{2} \tan (\varphi)\right)$ and finally along the line $y=\tan (\varphi) x$ from $\left(\frac{\pi^{2}}{2}, \frac{\pi^{2}}{2} \tan (\varphi)\right)$ to infinity.

Since $g_{4}(., u)$ is bounded on $\Gamma$, then

$$
\left|d^{4} \mathcal{H}(d, u)-\left(1-u^{2}\right) \tilde{\mathcal{H}}(d, u)\right| \leq K\left(1-u^{2}\right) e^{-\frac{\pi^{2}}{2 d}},
$$

where $K$ is positive constant. Finally

$$
d^{4} \mathcal{H}(d, u)=\left(1-u^{2}\right) \tilde{\mathcal{H}}(d, u)+\mathcal{O}\left(\left(1-u^{2}\right) e^{-\frac{\pi^{2}}{2 d}}\right) \quad \text { for } \quad\left(0<u_{0}<u \leq 1\right)
$$


2. We assume that $\mathcal{D}_{1}(d, u), \mathcal{D}_{2}(d, u)$ have property $\mathrm{G}$ and their first terms in the Taylor development at $d=0$ begin with $d^{k}$. Then

$$
\begin{aligned}
& \mathcal{D}_{1}=\int_{0}^{\infty} e^{-\frac{t}{d}} f(t, u) d t \\
& \mathcal{D}_{2}=\int_{0}^{\infty} e^{-\frac{t}{d}} g(t, u) d t
\end{aligned}
$$

where $f(t, u), g(t, u)$ are analytic in $|t|<\frac{\pi^{2}}{2}$ and $f(t, u)=O\left(t^{k-1}\right), g(t, u)=$ $O\left(t^{k-1}\right)$.

$$
\mathcal{D}_{1}(d, u) \mathcal{D}_{2}(d, u)=\int_{0}^{\infty} e^{-\frac{t}{d}}(f * g)(t, u) d t
$$

Since

$$
\begin{aligned}
h(t, u) & =(f * g)(t, u)=\int_{0}^{s} f(t, u) g(t-s, u) d s \\
& =\int_{0}^{t} f(t-s, u) g(s, u) d s \\
& =\int_{t / 2}^{t} f(s, u) g(t-s, u) d s+\int_{t / 2}^{t} f(t-s, u) g(s, u) d s .
\end{aligned}
$$

For $t<\frac{\pi^{2}}{2}$, the function $h(t, u)$ is $k$ times differentiable with respect to $t$ and

$$
\begin{aligned}
h^{\prime}(t, u) & =f(t, u) g(0, u)+f(0, u) g(t, u)-f\left(\frac{t}{2}, u\right) g\left(\frac{t}{2}, u\right) \\
& +\int_{t / 2}^{t} f(s, u) g^{\prime}(t-s, u) d s+\int_{t / 2}^{t} f^{\prime}(t-s, u) g(s, u) d s \\
& =\int_{t / 2}^{t} f(s) g^{\prime}(t-s) d s+\int_{t / 2}^{t} f^{\prime}(t-s, u) g(s, u) d s \\
& -f\left(\frac{t}{2}, u\right) g\left(\frac{t}{2}, u\right) \\
h^{(k)}(t, u) & =\int_{t / 2}^{t} f(s) g^{(k-1)}(t-s) d s+\int_{t / 2}^{t} f^{(k-1)}(t-s, u) g(s, u) d s \\
& -\sum_{n=0}^{k} f^{(n)}\left(\frac{t}{2}, u\right) g^{(k-1-n)}\left(\frac{t}{2}, u\right)
\end{aligned}
$$

Observe that $h^{(k)}(t, u)$ is continuous on $\left[0, \pi^{2}\right.$ [, it is analytic for $|t|<\frac{\pi^{2}}{2}$. If we put

$$
\tilde{h}(t, u)= \begin{cases}h(t, u), & \text { if } t<\frac{\pi^{2}}{2} \\ \left(t-\frac{\pi^{2}}{2}\right)^{k} \cdot & \text { if } t \geq \frac{\pi^{2}}{2}\end{cases}
$$


then

$$
\int_{0}^{\infty} e^{-\frac{t}{d} \tilde{h}^{(k)}}(t, u) d t
$$

has property $\mathrm{G}$ and

$$
\begin{aligned}
\left(\mathcal{D}_{1} \cdot \mathcal{D}_{2}\right)(d, u) & =\int_{0}^{\infty} e^{-\frac{t}{d}} h(t, u) d t=\int_{0}^{\infty} e^{-\frac{t}{d} \tilde{h}}(t, u) d t+\mathcal{O}\left(d^{k} e^{-\frac{\pi^{2}}{2 d}}\right) \\
& =d^{k} \int_{0}^{\infty} e^{-\frac{t}{d} \tilde{h}^{(k)}}(t, u) d t+\mathcal{O}\left(d^{k} e^{-\frac{\pi^{2}}{2 d}}\right) \\
& =d^{k} \mathcal{D}(d, u)+\mathcal{O}\left(d^{k} e^{-\frac{\pi^{2}}{2 d}}\right)
\end{aligned}
$$

where $\mathcal{D}(d, u)$ has property $\mathrm{G}$.

3. We have

$$
\mathcal{H}(d, u)^{2}=\frac{1}{d^{8}}\left(d^{4} \mathcal{H}\right) \cdot\left(d^{4} \mathcal{H}\right)
$$

Using (1) and (2) of this lemma, we obtain immediately the result.

For $0 \leq u \leq 1$, the proof of (4), (5), (6) and (7) is exactly the one of [5]. This proof is valid for $u_{0}<u \leq 1$.

\section{Approximate solution $\mathcal{A}(d, u)$ of the functional equa- tion (2.4)}

In this section we use the formal solution of (2.4). The estimates of section 5 for its coefficients and the preliminary results of section 6 allow to construct the function $\mathcal{A}(d, u)$ that almost satisfies (2.4), except an error which is exponentially small as $d \rightarrow 0$.

In theorem 2.1 we found that (2.4) has a uniquely determined formal power series solution

$$
A(d, u)=\frac{1}{\varepsilon} B(d, u)=\frac{1}{\varepsilon}\left(U+\sum_{\substack{n=7 \\ n \text { odd }}}^{\infty} F_{n}(u) d^{n}\right),
$$

where

$$
\begin{aligned}
U(d, u) & =\varepsilon u+\left(u-u^{3}\right) d^{3}+\left(\frac{10}{3} u^{5}-\frac{16}{3} u^{3}+2 u\right) d^{5}, \\
F_{n} & =\alpha n !\left(\frac{i}{\pi}\right)^{n+1} \tau_{n+1}^{\prime}(u)+\beta(n-2) !\left(\frac{i}{\pi}\right)^{n-1} \tau_{n-1}^{\prime}(u) \\
& +\gamma(n-4) !\left(\frac{i}{\pi}\right)^{n-4} \tau_{n-3}^{\prime}(u)+R_{n}(u), \\
\left\|R_{n}\right\|_{n} & =O\left((n-5) ! \pi^{-1} \log (n)\right), \quad(n \geq 7) .
\end{aligned}
$$


Consequently

$B(d, u)=\left(\alpha+\beta d^{2}+\gamma d^{4}\right) \sum_{\substack{n=7 \\ n \text { odd }}}^{\infty}(n+1) !\left(\frac{i}{\pi}\right)^{n+1} \frac{\tau_{n+2}(u)}{\tau_{2}(u)} d^{n}+\sum_{\substack{n=7 \\ n \text { odd }}}^{\infty} R_{n}(u) d^{n}+U(d, u)$,

Now we set

$\mathcal{B}(d, u)=\left(\alpha+\beta d^{2}+\gamma d^{4}\right) \frac{1}{\tau_{2}(u)} \mathcal{H}(d, u)+\mathcal{R}(d, u)+U(d, u),\left(d>0, u_{0}<u<1\right)$,

where $\mathcal{H}(d, u)$ is defined in (6.6) and $\tilde{\mathcal{R}}(d, u)$ is the function corresponding to $R_{n}, n=7,9, .$. By lemma 6.3 and proposition 6.2, we have

$$
\mathcal{B}(d, u) \sim B(d, u), \quad \text { as } d \searrow 0 \text { for every } \quad u_{0}<u<1 .
$$

Theorem 7.1. We have

$\left|\mathcal{A}\left(d, T^{+}\right)-\mathcal{A}\left(d, T^{-}\right)-f(\varepsilon, \mathcal{A}(d, u))\right| \leq \frac{K}{d} e^{-\frac{\pi^{2}}{2 d}}, \quad$ for $\left(0<d<d_{0}, u_{0} \leq u<1\right)$,

where $K$ is a constant independent of $d, u$ and

$$
\mathcal{A}(d, u)=\frac{1}{\varepsilon} \mathcal{B}(d, u)
$$

Proof. We set

$$
\mathcal{T}(d, u)=\mathcal{S}_{2}(\mathcal{B})-\varepsilon^{2}+\mathcal{B}(d, u)^{2}
$$

then

$$
\begin{aligned}
\mathcal{T}(d, u) & =\left(\alpha+\beta d^{2}+\gamma d^{4}\right) \mathcal{S}_{2}\left(\frac{1}{\tau_{2}(u)} \mathcal{H}\right)-\varepsilon^{2}+\mathcal{B}(d, u)^{2} \\
& +\frac{1}{2} \mathcal{R}\left(d, T^{+}\right)-\frac{1}{2} \mathcal{R}\left(d, T^{-}\right)+\mathcal{S}_{2}(U)
\end{aligned}
$$

With (7.2) and lemma 6.1, this implies

$$
\mathcal{T}(d, u)=\mathcal{T}_{0}(d, u) \cdot \mathcal{H}(d, u)+\mathcal{T}_{1}(d, u)+\mathcal{T}_{2}(d, u),
$$

where

$$
\begin{aligned}
\mathcal{T}_{0}(d, u) & =\left(\alpha+\beta d^{2}+\gamma d^{4}\right)[-2 \varepsilon u+2 U(d, u)] \frac{1}{\tau_{2}(u)}, \\
\mathcal{T}_{1}(d, u) & =\left(\alpha+\beta d^{2}+\gamma d^{4}\right)^{2} \frac{1}{\tau_{2}(u)^{2}} \mathcal{H}(d, u)^{2}+\mathcal{R}(d, u)^{2} \\
& +2\left(\alpha+\beta d^{2}+\gamma d^{4}\right) \frac{1}{\tau_{2}(u)} \mathcal{H}(d, u) \mathcal{R}(d, u) \\
\mathcal{T}_{2}(d, u) & =2 U(d, u) \mathcal{R}(d, u)+\frac{1}{2} \mathcal{R}\left(d, T^{+}\right)-\frac{1}{2} \mathcal{R}\left(d, T^{-}\right), \\
\mathcal{T}_{3}(d, u) & =U(d, u)^{2}+\mathcal{S}_{2}(U)-\varepsilon^{2}+\frac{d}{2} \mathcal{D}(d, u)
\end{aligned}
$$


Observing that $\mathcal{T}_{0}(d, u)$ is analytic function beginnings with $d^{3}$. Using (1) of lemma 6.6, we obtain that $d \mathcal{T}_{0}(d, u) \mathcal{H}(d, u)$ has property $\mathrm{G}$.

Applying lemma 6.6, we obtain

$$
\mathcal{T}_{1}(d, u)=d^{3} \mathcal{D}_{3}(d, u)+\mathcal{O}\left(d^{3} e^{-\frac{\pi^{2}}{2 d}}\right)
$$

where $\mathcal{D}_{3}(d, u)$ has property $\mathrm{G}$.

Using (5) of lemma 6.6 and theorem 6.4, we find

$$
\mathcal{T}_{2}(d, u)=d^{3} \mathcal{D}_{4}(d, u)+\mathcal{O}\left(d^{3} e^{-\frac{\pi^{2}}{2 d}}\right)
$$

where $\mathcal{D}_{4}(d, u)$ has property $\mathrm{G}$.

On the other hand, $\frac{1}{d} \mathcal{T}_{3}(d, u)$ has property $\mathrm{G}$. This implies

$$
\mathcal{T}(d, u)=\frac{1}{d} \mathcal{D}_{5}(d, u)+\mathcal{K}(d, u),
$$

where $\mathcal{D}_{5}(d, u)$ has property $\mathrm{G}$ and

$$
|\mathcal{K}(d, u)| \leq K d^{3} e^{\frac{-\pi^{2}}{2 d}} \quad \text { for } \quad\left(0<d<d_{0}, u_{0}<u<1\right) .
$$

Altogether we have proved that

$$
\mathcal{T}(d, u)=\frac{1}{d} \int_{0}^{\infty} e^{-\frac{t}{d}} \delta(t, u) d t+\mathcal{K}(d, u),
$$

where $\delta(., u)$ is analytic in $|t|<\frac{\pi^{2}}{2}$, it is continuous on $\left[0, \frac{\pi^{2}}{2}[\right.$ and $] \frac{\pi^{2}}{2}, \infty[$ and has a limit as $t \rightarrow \frac{\pi^{2}}{2}$ for every $u_{0}<u<1$ and satisfaies

$$
|\delta(t, u)| \leq K e^{K t}
$$

with a constant $K$ independent of $u$. Now if $\delta(t, u)=\sum_{n=0}^{\infty} \delta_{n}(t) t^{n}$ is the power series of $\delta(t, u)$ near $t=0$, Watson's lemma implies with (7.6) that

$$
d \mathcal{T}(d, u) \sim \sum_{n=0}^{\infty} n ! \delta_{n} d^{n+1}, \quad \text { as } d \searrow 0 \text { for every } u_{0}<u<1 .
$$

On the other hand, because of its definition

$$
\begin{aligned}
\mathcal{T}(d, u) & \sim \frac{1}{2} B\left(d, T^{+}\right)-\frac{1}{2} B\left(d, T^{-}\right)-\varepsilon^{2}+B(d, u)^{2} \\
& \sim 0+0 d+\cdots,
\end{aligned}
$$

because the formal series $B$ satisfy (5.1). But this means that all $\delta_{n}$ must vanish and $\delta(t, u) \equiv 0$ if

$|t|<\frac{\pi^{2}}{2}, u_{0}<u<1$. With (7.7), thus we obtain for $u_{0}<u<1$

$$
\int_{0}^{\infty} e^{-\frac{t}{d}}|\delta(t, u)| d t \leq \int_{\frac{\pi^{2}}{2}}^{\infty} e^{-\frac{t}{d}} K e^{K t} d t \leq K d e^{-\frac{\pi^{2}}{2 d}}, \quad\left(0<d<d_{0}\right) .
$$


We obtain

$$
|\mathcal{T}(d, u)| \leq K e^{-\frac{\pi^{2}}{2 d}} \quad\left(0<d<d_{0}\right) .
$$

Finally, we have shown that there is a constant $K$ such that

$$
\left|\mathcal{A}\left(d, T^{+}\right)-\mathcal{A}\left(d, T^{-}\right)-f(\varepsilon, \mathcal{A}(d, u))\right|=\frac{1}{\varepsilon}|\mathcal{T}(d, u)| \leq \frac{K}{d} e^{-\frac{\pi^{2}}{2 d}},
$$

for $0<d<d_{0}, 0 \leq u<1$.

\section{Distance between points of manifolds}

Let $y(t), t \in]-1, \infty[$ an exact solution to the difference equation (2.1), so that the stable manifold $W_{s}^{+}$is parametrized by $t \mapsto(y(t), y(t+\varepsilon))$. We know that $y^{-}(t)=-y^{-}(-t)$ is also an exact solution of (2.1) with $y^{-}(t) \rightarrow 1$ as $t \rightarrow-\infty$ . Morever the instable manifold $W_{i}^{-}$is parametrized by $t \mapsto\left(y^{-}(t), y^{-}(t+\varepsilon)\right)$. We introduced the quasi-solution $\mathcal{A}(d, u)$ in previous section, then $\mathcal{A}^{-}(d, u)=$ $-\mathcal{A}(d,-u)$ is a quasi-solution close to the exact solution $y^{-}(t)$. We denote by $\tilde{W}_{s}$, $\tilde{W}_{i}$ the manifolds close to $W_{s}^{+}$respectively $W_{i}^{-}$parametrized by $t \mapsto\left(\xi_{+}(t), \xi_{+}(t+\right.$ $\varepsilon)$ ) respectively $t \mapsto\left(\xi_{-}(t), \xi_{-}(t+\varepsilon)\right)$, where $\xi_{+}(t)=\mathcal{A}(d, u(t))$ and $\xi_{-}(t)=$ $\mathcal{A}^{-}(d, u(t))$.

In this section we will show that the vertical distance between a point of the stable manifold and the manifold $\tilde{W}_{s}$ is exponentially small as well as that between $\tilde{W}_{i}$ and $W_{i}^{-}$; both are smaller, as we will show than the distance between $\tilde{W}_{s}$ and $\tilde{W}_{i}$. This eventually proves that $W_{s}^{+}$and $W_{i}^{-}$do not coincide.

In order to estimate the distance between some point $\left(x_{0}, y_{0}\right)$ on the stable manifold $W_{s}^{+}$, we consider the sequence $P_{n}=\left(x_{n}, y_{n}\right)$, defined by $P_{n+1}=\phi\left(P_{n}\right)$, where

$$
\phi\left(\begin{array}{l}
x \\
y
\end{array}\right)=\left(\begin{array}{c}
y \\
x+2 \varepsilon\left(1-y^{2}\right)
\end{array}\right) .
$$

$P_{n}=\left(x_{n}, y_{n}\right)$ is a point of the stable manifold. We obtain

$$
P_{n+1}=\phi\left(\begin{array}{l}
x_{n} \\
y_{n}
\end{array}\right)=\left(\begin{array}{l}
x_{n+1} \\
y_{n+1}
\end{array}\right)=\left(\begin{array}{c}
y_{n} \\
x_{n}+2 \varepsilon\left(1-y_{n}^{2}\right)
\end{array}\right)
$$

Since $\Phi=\phi \circ \phi$, where $\Phi$ is defined in (1.3), and the stable manifold $W_{s}^{+}$is invariant by the application $\Phi$, then the stable manifold $W_{s}^{+}$remains invariant by $\phi$. Then theorem 7.1 implies

$$
\xi_{+}(t+\varepsilon)=\xi_{+}(t-\varepsilon)+2 \varepsilon\left(1-\xi_{+}(t)^{2}\right)+\frac{1}{\varepsilon} \eta(t) e^{-\frac{\pi^{2}}{2 \varepsilon}}
$$

where $\eta(t)$ is bounded. There is a sequence $\left.t_{n} \in\right]-1,+\infty[$ such that

$$
\xi_{+}\left(t_{n}-\varepsilon\right)=x_{n}=\mathcal{A}\left(d, T^{-}\left(d, u\left(t_{n}\right)\right)\right)=\mathcal{A}\left(d, T^{-}\left(d, U_{\varepsilon}(n)\right)\right)
$$




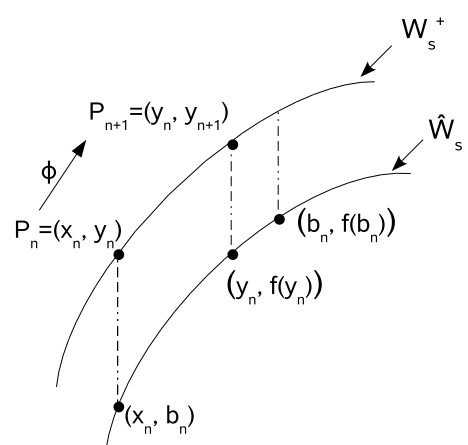

FIG. 4 - The vertical distance between a point of the stable manifold and the approximation defined using the quasi-solution $\mathcal{A}$ for the logistic equation .

$$
\begin{aligned}
\xi_{+}\left(t_{n}\right) & =\mathcal{A}\left(d, U_{\varepsilon}(n)\right) \\
\xi_{+}\left(t_{n}+\varepsilon\right) & =\mathcal{A}\left(d, T^{+}\left(d, U_{\varepsilon}(n)\right)\right)
\end{aligned}
$$

where $U_{\varepsilon}(n)=u\left(t_{n}\right)$. The relation (8.4) implies that the point $\left(x_{n}, b_{n}\right)$, where $b_{n}=$ $\xi_{+}\left(t_{n}\right)$, the vertical projection of the point $\left(x_{n}, y_{n}\right)$ on the curve $t \mapsto\left(\xi_{+}(t), \xi_{+}(t+\right.$ $\varepsilon))$. But, according to (8.2), 8.4), we have

$$
\xi_{+}\left(t_{n+1}-\varepsilon\right)=x_{n+1}=y_{n} .
$$

Now consider the error quantities

$$
d_{\varepsilon}(n)=y_{n}-\mathcal{A}\left(d, U_{\varepsilon}(n)\right)=y_{n}-b_{n},
$$

$d_{\varepsilon}(n)$ denotes the vertical distance between a point $P_{n}$ and the manifold $\hat{W}_{s}$. The manifold $\tilde{W}_{s}^{+}$is parameterized by the curve $t \mapsto\left(\xi_{+}(t), \xi_{+}(t+\varepsilon)\right)$. We obtain

$$
d_{\varepsilon}(n+1)=y_{n+1}-f\left(x_{n+1}\right)=y_{n+1}-f\left(y_{n}\right)=y_{n+1}-f\left(d_{\varepsilon}(n)+b_{n}\right),
$$

where $f(x)=\xi_{+}\left(\xi_{+}^{-1}(x)+\varepsilon\right)$. The point $\left(y_{n}, f\left(y_{n}\right)\right)$ is the vertical projection of the point $\left(x_{n+1}, y_{n+1}\right)$ on the curve $t \mapsto\left(\xi_{+}(t), \xi_{+}(t+\varepsilon)\right) \quad$ (See FIG.4).

If we use Taylor expainson, (8.8) implies

$$
d_{\varepsilon}(n+1)=y_{n+1}-f\left(b_{n}\right)-f^{\prime}\left(\theta_{n}\right) d_{\varepsilon}(n), \quad b_{n}<\theta_{n}<b_{n}+d_{\varepsilon}(n) .
$$

Then according to the definitions of $f$ and $b_{n}$, we have $f\left(b_{n}\right)=\xi_{+}\left(t_{n}+\varepsilon\right)$, with (8.3) and (8.4), we obtain

$$
f\left(b_{n}\right)=x_{n}+2 \varepsilon\left(1-b_{n}^{2}\right)+\frac{1}{\varepsilon} \eta\left(t_{n}\right) e^{-\frac{\pi^{2}}{2 \varepsilon}} .
$$

Together with (8.9) and (8.2) this implies 


$$
d_{\varepsilon}(n+1)=-f^{\prime}\left(\theta_{n}\right) d_{\varepsilon}(n)+2 \varepsilon\left(b_{n}^{2}-y_{n}^{2}\right)-\frac{1}{\varepsilon} \eta\left(t_{n}\right) e^{-\frac{\pi^{2}}{2 \varepsilon}},
$$

Using (8.7) we obtain

$$
d_{\varepsilon}(n+1)=-\left(f^{\prime}\left(\theta_{n}\right)+2 \varepsilon\left(y_{n}+b_{n}\right)\right) d_{\varepsilon}(n)-\frac{1}{\varepsilon} \eta\left(t_{n}\right) e^{-\frac{\pi^{2}}{2 \varepsilon}} .
$$

Now

$$
f^{\prime}\left(\theta_{n}\right)=\frac{\xi_{+}^{\prime}\left(z_{n}+\varepsilon\right)}{\xi_{+}^{\prime}\left(z_{n}\right)}, \quad \text { where } \quad z_{n}=\xi_{+}^{-1}\left(\theta_{n}\right)
$$

then

$$
f^{\prime}\left(\theta_{n}\right)=1-2 u\left(z_{n}\right) d+\left(3 u\left(z_{n}\right)^{2}-1\right) d^{2}+O\left(d^{3}\right) .
$$

On the other hand $b_{n}<\theta_{n}<y_{n}$ implies

$$
0<b_{n}<\xi\left(z_{n}\right)<y_{n}
$$

and thus

$$
\begin{aligned}
f^{\prime}\left(\theta_{n}\right)+2 \varepsilon\left(y_{n}+b_{n}\right) & >1+2 \varepsilon\left(\xi_{+}\left(z_{n}\right)+b_{n}\right)-2 u\left(z_{n}\right) d \\
& +\left(3 u\left(z_{n}\right)^{2}-1\right) d^{2}+O\left(d^{3}\right) .
\end{aligned}
$$

We know that

$$
\xi_{+}\left(z_{n}\right)=u\left(z_{n}\right)+\left(u\left(z_{n}\right)-u\left(z_{n}\right)^{3}\right) d^{2}+O\left(d^{4}\right)
$$

this with (2.8) implies

$$
f^{\prime}\left(\theta_{n}\right)+2 \varepsilon\left(y_{n}+b_{n}\right)>1+2 \varepsilon b_{n}+O\left(d^{2}\right) .
$$

As $b_{n}>0$, then for sufficiently small $d$,

$$
f^{\prime}\left(\theta_{n}\right)+2 \varepsilon\left(y_{n}+b_{n}\right)>1 .
$$

This implies

$$
f^{\prime}\left(\theta_{n}\right)+2 \varepsilon\left(y_{n}+b_{n}\right)=1+g_{\varepsilon}\left(u\left(z_{n}\right)\right), \quad \text { with some } g_{\varepsilon}\left(u\left(z_{n}\right)\right) \geq \varepsilon c,
$$

$g_{\varepsilon}\left(u\left(z_{n}\right)\right)=O(\varepsilon)$ and $c$ is a positive constant. With (8.12), this implies

$$
d_{\varepsilon}(n+1)=-\left(1+g_{\varepsilon}\left(u\left(z_{n}\right)\right)\right) d_{\varepsilon}(n)-\frac{1}{\varepsilon} \eta\left(t_{n}\right) e^{-\frac{\pi^{2}}{2 \varepsilon}} .
$$

As $d_{\varepsilon}(n) \rightarrow 0$ as $n \rightarrow+\infty$, this implies that

$$
\left|d_{\varepsilon}(n)\right| \leq \frac{1}{\varepsilon} e^{-\frac{\pi^{2}}{2 \varepsilon}} \sum_{k=n}^{\infty}\left|\frac{\eta\left(s_{k}\right)}{\prod_{i}^{i=k}\left(1+g_{\varepsilon}\left(u\left(z_{k}\right)\right)\right)}\right|,
$$


provided this series converges. This follows from

$$
\sum_{k=n}^{\infty}\left|\frac{\eta\left(s_{k}\right)}{\prod_{i}^{i=k}\left(1+g_{\varepsilon}\left(u\left(z_{k}\right)\right)\right)}\right| \leq K_{1} \sum_{k=0}^{+\infty}(1+\varepsilon c)^{-k},
$$

where we used that $\eta$ is bounded. This imply that there is a constant $K>0$ such that

$$
\sum_{k=n}^{\infty}\left|\frac{\eta\left(s_{k}\right)}{\prod_{i}^{i=k}\left(1+g_{\varepsilon}\left(u\left(z_{k}\right)\right)\right)}\right| \leq \frac{K}{\varepsilon} .
$$

With (8.5) implies

$$
d_{\varepsilon}(n)=O\left(\frac{1}{\varepsilon^{2}} \exp \left(-\frac{\pi^{2}}{2 \varepsilon}\right)\right)
$$

and in particular that the vertical distance $d_{\varepsilon}(0)$ of $\left(x_{0}, y_{0}\right)$ to $\tilde{W}_{s}$ is

$$
d_{\varepsilon}(0)=O\left(\frac{1}{\varepsilon^{2}} \exp \left(-\frac{\pi^{2}}{2 \varepsilon}\right)\right) .
$$

Now we will calculate the vertical distance between the twomanifolds $\tilde{W}_{s}$ and $\tilde{W}_{i}$. To do this, we will need to extend both quasi-solutions in an adequate way. We define

$$
\begin{aligned}
& \tilde{\xi}_{+}(t)=\mathcal{A}(d, u(t)), \quad \text { for } \quad-\frac{1}{2} \leq u \leq 1, \\
& \tilde{\xi}_{-}(t)=\mathcal{A}^{-}(d, u(t)), \text { for } \quad-1 \leq u \leq \frac{1}{2} .
\end{aligned}
$$

First, we evaluate the quantities

$$
d_{\varepsilon}\left(\tilde{\xi}_{+}(t), \tilde{\xi}_{-}(t)\right)=\mathcal{A}(d, u)-\mathcal{A}^{-}(d, u), \text { for }-\frac{1}{2} \leq u \leq \frac{1}{2}
$$

using (7.2) and (7.4)

$$
\begin{aligned}
d_{\varepsilon}\left(\tilde{\xi}_{+}(t), \tilde{\xi}_{-}(t)\right) & =\tilde{\xi}_{+}(t)-\tilde{\xi}_{-}(t) \\
& =\frac{1}{\varepsilon}\left(\alpha+\beta d^{2}+\gamma d^{4}\right)\left(\tilde{\mathcal{H}}(d, u)-\tilde{\mathcal{H}}^{-}(d, u)\right)
\end{aligned}
$$

where

$$
\begin{aligned}
\tilde{\mathcal{H}}(d, u) & =\int_{0}^{\infty} e^{-\frac{s}{d}} h(s, u) d s, \quad \text { for } \quad-\frac{1}{2} \leq u \leq \frac{1}{2}, \\
\tilde{\mathcal{H}}^{-}(d, u) & =-\int_{0}^{\infty} e^{-\frac{s}{d}} h(s,-u) d s, \quad \text { for } \quad-\frac{1}{2} \leq u \leq \frac{1}{2} .
\end{aligned}
$$


Modifying the path of integral, we obtain

$$
\begin{aligned}
\tilde{\mathcal{H}}(d, u)=\int_{\Gamma} e^{-\frac{s}{d}} h(s, u) d s & +\sum_{\operatorname{Im}\left(s_{k}(u)\right)<0} 2 \pi i \operatorname{Res}\left(e^{-\frac{s}{d}} h(s, u), s_{k}(t)\right) \\
& -\sum_{\operatorname{Im}\left(s_{k}(u)\right)>0} 2 \pi i \operatorname{Res}\left(e^{-\frac{s}{d}} h(s, u), s_{k}(t)\right),
\end{aligned}
$$

where $s_{k}(t)=\frac{\pi^{2}}{2} \pm \frac{d \pi t}{\varepsilon} i+k \pi^{2}$ for $k \geq 0, \Gamma$ is the sum of two paths $\Gamma_{1}$ and $\Gamma_{2}$. Here $\Gamma_{1}$ consist of two segments of the higher half-plane, one of these two segments is parallel to the axis $y=0$ and begins at the point $\left(\frac{\pi^{2}}{4}, 1\right)$, the other ends in the point $(0,0)$, and the path $\Gamma_{2}$ is the symmetry of $\Gamma_{1}$ relatively to the axis $y=0$.

This implies

$$
\begin{aligned}
\tilde{\mathcal{H}}(d, u)=\int_{\Gamma} e^{-\frac{s}{d}} h(s, u) d s & +\frac{\pi e^{\frac{\pi t}{\varepsilon} i}}{2 d^{2}\left(1-u^{2}\right)} \sum_{k=0}^{\infty} \exp \left(-\frac{(k+1) \pi^{2}}{2 d}\right) \\
& +\frac{\pi e^{-\frac{\pi t}{\varepsilon} i}}{2 d^{2}\left(1-u^{2}\right)} \sum_{k=0}^{\infty} \exp \left(-\frac{(k+1) \pi^{2}}{2 d}\right)
\end{aligned}
$$

Therefore

$$
\tilde{\mathcal{H}}(d, u)=\int_{\Gamma} e^{-\frac{s}{d}} h(s, u) d s+\frac{\pi \cos \left(\frac{\pi t}{\varepsilon}\right)}{d^{2}\left(1-u^{2}\right)} \sum_{k=0}^{\infty} \exp \left(-\frac{(k+1) \pi^{2}}{2 d}\right) .
$$

Similary for $\tilde{\mathcal{H}}^{-}(d, u)$

$$
\tilde{\mathcal{H}}^{-}(d, u)=\int_{\Gamma} e^{-\frac{s}{d}} h(s, u) d s-\frac{\pi \cos \left(\frac{\pi t}{\varepsilon}\right)}{d^{2}\left(1-u^{2}\right)} \sum_{k=0}^{\infty} \exp \left(-\frac{(k+1) \pi^{2}}{2 d}\right) .
$$

Therefore

$d_{\varepsilon}\left(\tilde{\xi}_{+}(t), \tilde{\xi}_{-}(t)\right)=\frac{2 \pi\left(\alpha+\beta d^{2}+\gamma d^{4}\right) \cos \left(\frac{\pi t}{\varepsilon}\right)}{\varepsilon^{3}\left(1-u^{2}\right)} \exp \left(-\frac{\pi^{2}}{2 d}\right)+\mathcal{O}\left(\frac{\cos \left(\frac{\pi t}{\varepsilon}\right)}{\varepsilon^{3}} e^{-\frac{3 \pi^{2}}{2 d}}\right)$, for $-\operatorname{arctanh}\left(\frac{1}{2}\right)<t<\operatorname{arctanh}\left(\frac{1}{2}\right)$. Consequently

$$
d_{\varepsilon}\left(\tilde{\xi}_{+}(t), \tilde{\xi}_{-}(t)\right)=\frac{2 \pi \alpha \cos \left(\frac{\pi t}{\varepsilon}\right)}{\varepsilon^{3}\left(1-\tanh \left(\frac{d}{\varepsilon} t\right)^{2}\right)} e^{-\frac{\pi^{2}}{2 \varepsilon}}+\mathcal{O}\left(\frac{1}{\varepsilon} \cos \left(\frac{\pi t}{\varepsilon}\right) e^{-\frac{\pi^{2}}{2 d}}\right)
$$

Now we want to estimate the vertical distance between a point on the quasimanifold $\tilde{W}_{s}$ and its vertical projection on the quasimanifold $\tilde{W}_{i}$. For this purpose let $t$ such that $-\frac{1}{2} \leq u(t) \leq \frac{1}{2}$. Then the point $\left(\tilde{\xi}_{+}(t), \tilde{\xi}_{+}(t+\varepsilon)\right)$ is on the quasimanifold $\tilde{W}_{s}$. We suppose that $\left(\tilde{\xi}_{+}(t), \tilde{\xi}^{-}\left(t_{1}+\varepsilon\right)\right)$, where $-\frac{1}{2} \leq u\left(t_{1}\right) \leq \frac{1}{2}$, is its 
vertical projection on the quasimanifold $\tilde{W}_{i}$, then the vertical distance between these two points is

$$
\operatorname{dist}_{v}(t)=\tilde{\xi}_{+}(t+\varepsilon)-\tilde{\xi}_{-}\left(t_{1}+\varepsilon\right)=\tilde{\xi}_{+}(t+\varepsilon)-\tilde{f}\left(\tilde{\xi}_{+}(t)\right),
$$

where $\tilde{f}(x)=\tilde{\xi}_{-}\left(\tilde{\xi}_{-}^{-1}(x)+\varepsilon\right), \tilde{\xi}_{-}^{-1}$ the inverse function of $\tilde{\xi}_{-}$. Using (8.22) and (8.20) we obtain

$$
\operatorname{dist}_{v}(t)=\tilde{\xi}_{+}(t+\varepsilon)-\tilde{f}\left(\tilde{\xi}_{-}(t)+e_{\varepsilon}(t)\right),
$$

where $e_{\varepsilon}(t)=d_{\varepsilon}\left(\tilde{\xi}_{+}(t), \tilde{\xi}_{-}(t)\right)$. We use Taylor expansion and obtain

$$
\begin{aligned}
\operatorname{dist}_{v}(t) & =\tilde{\xi}_{+}(t+\varepsilon)-\tilde{f}\left(\tilde{\xi}_{-}(t)\right)-e_{\varepsilon}(t) \cdot \tilde{f}^{\prime}(\theta), \\
& =\tilde{\xi}_{+}(t+\varepsilon)-\tilde{\xi}_{-}(t+\varepsilon)-e_{\varepsilon}(t) \cdot \tilde{f}^{\prime}(\theta),
\end{aligned}
$$

where $\tilde{\xi}_{-}(t)<\theta<\tilde{\xi}_{-}(t)+e_{\varepsilon}(t)$. With 8.20$)$ this implies

$$
\operatorname{dist}_{v}(t)=e_{\varepsilon}(t+\varepsilon)-e_{\varepsilon}(t) \cdot \tilde{f}^{\prime}(\theta) .
$$

Since $e_{\varepsilon}(t+\varepsilon)=-e_{\varepsilon}(t)+\mathcal{O}\left(\varepsilon \cdot e_{\varepsilon}(t)\right)$ and $\tilde{f}^{\prime}(\theta)=\frac{\tilde{\xi}_{-}^{\prime}\left(\tilde{\xi}_{-}^{-1}(\theta)+\varepsilon\right)}{\tilde{\xi}_{-}^{\prime}\left(\tilde{\xi}_{-}^{-1}(\theta)\right)}=1+\mathcal{O}(\varepsilon)$, we obtain

$$
\operatorname{dist}_{v}(t)=(-2+\mathcal{O}(\varepsilon)) e_{\varepsilon}(t)
$$

Consequently

$$
\operatorname{dist}_{v}(t) \sim-\frac{4 \pi \alpha \cos \left(\frac{\pi t}{\varepsilon}\right)}{\varepsilon^{3}\left(1-\tanh \left(\frac{d}{\varepsilon} t\right)^{2}\right)} e^{-\frac{\pi^{2}}{2 \varepsilon}} .
$$

Combining (8.16) and (8.27), we conclude

$$
\operatorname{Dist}_{\varepsilon}\left(w_{s}^{+}(t), W_{i}^{-}\right)=\frac{4 \pi \alpha \cos \left(\frac{\pi}{\varepsilon} t+\pi\right)}{\varepsilon^{3}\left(1-\tanh \left(\frac{d}{\varepsilon} t\right)\right)} e^{\frac{-\pi^{2}}{2 \varepsilon}}+O\left(\frac{1}{\varepsilon^{2}} e^{\frac{-\pi^{2}}{2 \varepsilon}}\right), \quad \text { as } \varepsilon \searrow 0,
$$

\section{An estimate for the main asymptotic coefficients}

In this section we show that the number $\alpha$ in the main theorem of the introduction is not zero and we want to find bounds for $\alpha_{n}$ and will then obtain estimats for $\alpha$ using the first $\alpha_{n}$ which can be computed explicitly. We recall that

$$
\alpha=\frac{1}{\pi} \sum_{n=7}^{\infty} \alpha_{n}
$$


where $\alpha_{n}(n-1) !\left(\frac{i}{\pi}\right)^{n-1} u^{n}$ is the leading term of the coefficient $\{\mathcal{C}(G)\}_{n}(u)$ of $d^{n}$ in $\mathcal{C}(G)(d, u)$, where $G=Q(d, u) \cdot F(d, u), F(d, u)=B(d, u)-U(d, u)$ (cf.(5.2) and $(5.5))$.

For a polynomial series $X(d, u)=\sum_{n=0}^{\infty} X_{n}(u) d^{n}$ where the degrees of $X_{n}(u)$ do not exceed $n$, we can write $X_{n}(u)=\sum_{k=0}^{n} x_{n k} u^{n}$. Then we denote by $\widehat{X}(z)=$ $\sum_{n=0}^{\infty} x_{n n} z^{n}$.

The mapping $X(d, u) \mapsto \widehat{X}(z)$ extracts the leading terms of the series $X(d, u)$. It is compatible with addition and multiplication. If we define the following operator $\widehat{D}=-z^{2} \frac{d}{d z}$, we also find

$$
\begin{aligned}
& \widehat{D} \widehat{X}(z)=\widehat{D X}(z)=-z^{2} \widehat{X}^{\prime}(z) \\
& \widehat{\mathcal{C}(X)}(z)=\cosh \left(\frac{\widehat{D}}{2}\right) \widehat{X}(z):=\frac{1}{2}\left(\widehat{X}\left(\frac{2 z}{2+z}\right)+\widehat{X}\left(\frac{2 z}{2-z}\right)\right) \\
& \widehat{\mathcal{S}(X)}(z)=\sinh \left(\frac{\widehat{D}}{2}\right) \widehat{X}(z):=\frac{1}{2}\left(\widehat{X}\left(\frac{2 z}{2+z}\right)-\widehat{X}\left(\frac{2 z}{2-z}\right)\right) \\
& \widehat{\mathcal{C}_{2}(X)}(z)=\cosh (\widehat{D}) \widehat{X}(z):=\frac{1}{2}\left(\widehat{X}\left(\frac{z}{1+z}\right)+\widehat{X}\left(\frac{z}{1-z}\right)\right) \\
& \widehat{\mathcal{S}_{2}(X)}(z)=\sinh (\widehat{D}) \widehat{X}(z):=\frac{1}{2}\left(\widehat{X}\left(\frac{z}{1+z}\right)-\widehat{X}\left(\frac{z}{1-z}\right)\right)
\end{aligned}
$$

where the operators $\mathcal{S}, \mathcal{C}, \mathcal{S}_{2}, \mathcal{C}_{2}$ are defined in (2.18). If we apply the mapping $X(d, u) \mapsto \widehat{X}(z)$ to the equation (5.13), we obtain

$$
\widehat{\mathcal{L}}\left(\frac{\widehat{E}}{\widehat{Q}_{1}}\right)(z)=\widehat{\mathcal{R}}(\widehat{E})(z),
$$


where

$$
\begin{aligned}
& \widehat{\mathcal{L}}(x):=-\frac{\widehat{V}_{1}}{z^{2}}\left(\widehat{\mathcal{S}}(x)-\frac{\widehat{\mathcal{S}}\left(z^{2}\right)}{\widehat{\mathcal{C}}\left(z^{2}\right)} \widehat{\mathcal{C}}(x)\right) \\
& \widehat{\mathcal{R}}(x):=-\frac{\widehat{\mu}}{z^{2}}+\frac{\widehat{W}_{5}}{z^{2}} \widehat{\mathcal{C}}\left(\frac{x}{\widehat{Q}_{1}}\right)-\frac{\widehat{W}_{1}}{2 z^{2} \widehat{Q}} \mathcal{Y}+\frac{\widehat{W}_{4}}{2 z^{2} \widehat{Q}^{2}} \widehat{\mathcal{C}}\left(\frac{x}{\widehat{Q}_{1}}\right) \mathcal{Y}^{2} \\
& -\frac{\widehat{W}_{2}}{2 z^{2}} \widehat{\mathcal{C}}_{2}\left(\frac{\mathcal{Y}}{\widehat{Q}}\right) \\
& \widehat{E}=\widehat{\mathcal{C}(G)}=\sum_{n=0 \text { odd }}^{\infty} \alpha_{n}(n-1) !\left(\frac{i}{\pi}\right)^{n-1} z^{n}, \\
& \mathcal{Y}=\widehat{\mathcal{C}}^{-1}(x), \\
& \widehat{Q}=-z^{2}+z^{4}-\frac{13}{6} z^{6}+\frac{47}{18} z^{8}, \\
& \widehat{Q}_{1}=-z^{2}-\frac{3}{2} z^{4}, \\
& \widehat{V}_{1}=4 z^{2}\left[-192-240 z^{2}+508 z^{4}+1995 z^{6}+3418 z^{8}\right. \\
& \left.+\quad 4389 z^{10}+217 z^{12}\right] /\left[3\left(z^{2}-4\right)^{4}\right], \\
& \widehat{W}_{2}=z^{11}\left[591-15457 z^{2}+21610 z^{4}+59194 z^{6}+36583 z^{8}\right. \\
& \left.+2759 z^{10}\right] /\left[27\left(z^{2}-1\right)^{8}\right] \\
& \widehat{W}_{1}=z^{11}\left[2385-32832 z^{2}-11790 z^{4}+231917 z^{6}-116267 z^{8}+720682 z^{10}\right. \\
& \left.+\quad 1018936 z^{12}+290089 z^{14}+2480 z^{16}\right] /\left[81\left(z^{2}-1\right)^{8}\right] \\
& \widehat{W}_{4}=\widehat{V} \widehat{\mathcal{C}}_{2}(\widehat{Q})+\widehat{W} \widehat{\mathcal{S}}_{2}(\widehat{Q}) \\
& =-z^{2}\left[54-432 z^{2}+1449 z^{4}-2724 z^{6}+4449 z^{8}-14719 z^{10}-12857 z^{12}\right. \\
& \left.+95146 z^{14}+110905 z^{16}+29041 z^{18}+248 z^{20}\right] /\left[54\left(z^{2}-1\right)^{8}\right], \\
& \widehat{W}_{5}=\widehat{W}_{3}+\widehat{P} \widehat{V}_{3}, \\
& =-8 z^{5}\left[-1152+1168 z^{2}+9744 z^{4}+18901 z^{6}+14664 z^{8}\right. \\
& \left.+\quad 1240 z^{10}\right] /\left[3\left(z^{2}-4\right)^{4}\left(4+z^{2}\right)\right],
\end{aligned}
$$




$$
\begin{aligned}
\widehat{\mu} & =z^{10}\left[-527000 z^{30}-1988300 z^{28}+14763570 z^{26}-25488145 z^{24}\right. \\
& +11808105 z^{22}+11381027 z^{20}-17065562 z^{18}-15479497 z^{16}-15777878 z^{14} \\
& -21499321 z^{12}+14844222 z^{10}+4847178 z^{8}-1419003 z^{6}-541026 z^{4} \\
& \left.+320490 z^{2}-50220\right] /\left[972\left(z^{2}-1\right)^{12}\right]
\end{aligned}
$$

with $x=x(z)$ is a power series.

Now for integers $m>p \geq-1, m \geq 0$, we introduce the set

$$
\mathcal{M}(m, p)=\left\{\sum_{n=m}^{\infty}{ }^{*} x_{n} z^{n} \backslash x_{n} /\left[(n-p) ! \pi^{-n}\right], n=m, m+2 \ldots \text { is bounded }\right\}
$$

$\mathcal{M}(m, p)$ is a Banach space with the norm

$$
|x|_{m, p}=\sup \left\{\left|x_{n}\right| /\left[(n-p) ! \pi^{-n}\right], n \geqq m, n \equiv m \quad \bmod 2\right\} .
$$

Here the symbol $\sum_{n=m}^{\infty}{ }^{*}$ for the summation means that only summands with $n \equiv m \bmod 2$ are considered.

Proposition 9.1. Let $m, p, q$ be integers and $m \geq p \geq-1, m \geq q \geq-1, m \geq 0$. The norms (9.4) have the following property:

1. If $q<p \leq m$ then $\mathcal{M}(m, p) \subset \mathcal{M}(m, q)$ and

$$
|x|_{m, q} \leq \frac{(m-p) !}{(m-q) !}|x|_{m, p} \quad \text { for } x \in \mathcal{M}(m, p)
$$

2. If $x \in \mathcal{M}(m, p)$ then $z x \in \mathcal{M}(m+1, p+1)$ and

$$
|z x|_{m+1, p+1}=\pi|x|_{m, p}
$$

3. $\widehat{D}: \mathcal{M}(m, p) \mapsto \mathcal{M}(m+1, p)$ and

$$
\pi|x|_{m, p} \leq|\widehat{D}(x)|_{m+1, p} \leq \pi \frac{m}{m-p+1}|x|_{m, p}
$$

Now we consider an even power series $f(z)=\sum_{j=0}^{\infty} * f_{j} z^{j}$ having a radius of convergence greater than $\pi$. Then $f(\widehat{D})$ maps $\mathcal{M}(m, p)$ into itself and

$$
|f(\widehat{D}) x|_{m, p} \leq\|f\|_{m, p}|x|_{m, p}, \quad \text { for } x \in \mathcal{M}(m, p)
$$

where

$$
\begin{aligned}
& \|f\|_{m, p}=\sup \left\{\sum_{j=0}^{n-m}{ }^{*}\left|f_{j}\right| \pi^{j} \prod_{k=1}^{p-1} \frac{n-k}{n-j-k} \backslash n \geq m, n \equiv m \quad \bmod 2\right\} \text { if } p \geq 1, \\
& \|f\|_{m, p}=\sup \left\{\sum_{j=0}^{\infty}{ }^{*}\left|f_{j}\right| \pi^{j} \backslash n \geq m, n \equiv m \quad \bmod 2\right\} \text { if } p \leq 0 .
\end{aligned}
$$


We have also

$$
|f . x|_{m, p} \leq M_{m, p}(f)|x|_{m, p}, \quad \text { for } x \in \mathcal{M}(m, p),
$$

where

$$
M_{m, p}(f)=\sup \left\{\sum_{j=0}^{n-m}\left|f_{j}\right| \pi^{j} \frac{(n-p-j) !}{(n-p) !} \backslash n \geq m, n \equiv m \quad \bmod 2\right\} .
$$

We conclude that the inverse of $\widehat{\mathcal{C}}$ maps $\mathcal{M}(m, p)$ with $p \geq 3$ into $\mathcal{M}(m, 1)$ and

$$
\left|\widehat{\mathcal{C}}^{-1}(x)\right|_{m, 1} \leq c_{m, p}|x|_{m, p}, \text { with } c_{m, p}=\frac{4}{\pi} \sum_{n=m}^{\infty} * \frac{(n-p) !}{(n-1) !} .
$$

Let $x \in \mathcal{M}(m, p)$ and $y \in \mathcal{M}(m, q)$ and assume that $m+q \leq n+p$. Then we obtain $x . y \in \mathcal{M}(n+m, q+m)$ and

$$
|x . y|_{n+m, q+m} \leq \alpha_{m-p, n-q}|x|_{m, p}|y|_{n, q},
$$

with

$$
\alpha_{r, s}=\sup \left\{\sum_{j=r}^{N-s} * \frac{j !(N-j) !}{(N-r) !} \backslash N \geq r+s, N-r-s \text { even }\right\} .
$$

Finally we need estimates for the inverse of the linear operator $\widehat{\mathcal{L}}$ in $(9.2) . \widehat{\mathcal{L}}$ maps $\mathcal{M}(m-1, q)$ onto $\mathcal{M}(m, q)$ and is one to one.Now let $x \in \mathcal{M}(m-1, p)$ and $w=\widehat{\mathcal{L}}(x)$. If we proceed similar to the proof of theorem 4.6, we find that

$$
\begin{aligned}
\widehat{D} y & =f(\widehat{D})\left[-\frac{\left(4+z^{2}\right)}{4 \widehat{V}_{1}} w\right], \\
x & =z^{2} y .
\end{aligned}
$$

where $f(z)=z / \sinh (z / 2)$.

We can apply (9.6)..(9.13) and obtain for $m \geq \max (p+1,4)$

$$
\left|\widehat{\mathcal{L}}^{-1}(w)\right|_{m-1, p} \leq L_{m, p}|w|_{m, p} \quad \text { for } w \in \mathcal{M}(m, p),
$$

where

$$
L_{m, p}=\frac{1}{\pi}\left\|\frac{z}{\sinh (z / 2)}\right\|_{m-2, p-2} M_{m, p}\left(\frac{\left(4+z^{2}\right) z^{2}}{4 \widehat{V}_{1}}\right) .
$$

Now we are in a position to use (9.2) to estimate $\widehat{E}$. We use that $\widehat{E}$ can be computed recursively from (9.2). If $x$ is an odd power series and $x \equiv \widehat{E} \bmod z^{n}$ with $n \geq 9$ then via $\widehat{\mathcal{C}}^{-1}(x) \equiv \widehat{G} \bmod z^{n}$ we first obtain $\widehat{\mathcal{R}}(x) \equiv \widehat{\mathcal{R}}(\widehat{E}) \bmod z^{n+1}$ 
and then $\widehat{\mathcal{L}}^{-1}(\widehat{\mathcal{R}}(x)) \equiv \frac{\widehat{E}}{\widehat{Q}_{1}} \bmod z^{n+1}$. Thus if $x_{0} \equiv 0 \bmod z^{9}$ is arbitrary and we let

$$
x_{n+1}:=\widehat{Q}_{1} \widehat{\mathcal{L}}^{-1}\left(\widehat{\mathcal{R}}\left(x_{n}\right)\right) \text { for } n=0 \ldots
$$

then $x_{n} \equiv \widehat{E} \bmod z^{9+2 n}$ for all $n$. We begin with the polynomial $x_{0}$ of degree $\leq 79$ with $x_{0} \equiv \widehat{E} \bmod z^{81}$. We computed it explicitly and found that

$$
\left|x_{0}\right|_{9,7} \leq K_{0}:=308027.359777894414
$$

Then we consider the sequence $x_{n}$ of polynomial series defined in (9.17), which now satisfies $x_{n} \equiv \widehat{E} \bmod z^{81+2 n}$. We will show

$$
\left|x_{n}\right|_{9,7} \leq K_{0} \quad \text { for all } n \geq 0
$$

and this implies $|\widehat{E}|_{9,7} \leq K_{0}$ because each coefficient of $\widehat{E}$ is also that of some $x_{n}$ if $n$ is sufficiently large.

We prove (9.19) by induction. For $n=0$ it is true. Assume that $\left|x_{n}\right|_{9,7} \leq K_{0}$, for this Let us take again the equation (9.18) with (9.2). We obtain

$$
\begin{aligned}
x_{n+1} & +\widehat{Q}_{1} \widehat{\mathcal{L}}^{-1}\left(\frac{\widehat{\mu}}{z^{2}}\right)=\widehat{Q}_{1} \widehat{\mathcal{L}}^{-1}\left(\frac{\widehat{W}_{5}}{z^{2}} \widehat{\mathcal{C}}\left(\frac{x_{n}}{\widehat{Q}_{1}}\right)\right)-\widehat{Q}_{1} \widehat{\mathcal{L}}^{-1}\left(\frac{\widehat{W}_{1}}{2 z^{2}} \widehat{\mathcal{Y}}_{n}\right) \\
& +\widehat{Q}_{1} \widehat{\mathcal{L}}^{-1}\left(\frac{\widehat{W}_{4}}{2 z^{2} \widehat{Q}^{2}} \widehat{\mathcal{C}}\left(\frac{x_{n}}{\widehat{Q}_{1}}\right) \mathcal{Y}_{n}^{2}\right)-\widehat{Q}_{1} \widehat{\mathcal{L}}^{-1}\left(\frac{\widehat{W}_{2}}{2 z^{2}} \widehat{\mathcal{C}}_{2}\left(\frac{\mathcal{Y}_{n}}{\widehat{Q}}\right)\right)
\end{aligned}
$$

where $\mathcal{Y}_{n}=\widehat{\mathcal{C}}^{-1}\left(x_{n}\right)$. Then using (9.2)..(9.17), we obtain

$$
\begin{array}{r}
\left|\widehat{Q}_{1} \widehat{\mathcal{L}}^{-1}\left(\frac{\widehat{W}_{5}}{z^{2}} \widehat{\mathcal{C}}\left(\frac{x_{n}}{\widehat{Q}_{1}}\right)\right)\right|_{11,10} \leq K_{1}\left|x_{0}\right|_{9,7}, \\
\left|\widehat{Q}_{1} \widehat{\mathcal{L}}^{-1}\left(\frac{\widehat{W}_{1}}{2 z^{2}} \mathcal{Y}_{n}\right)\right|_{17,10} \leq K_{2}\left|x_{0}\right|_{9,7}, \\
\left|\widehat{Q}_{1} \widehat{\mathcal{L}}^{-1}\left(\frac{\widehat{W}_{2}}{2 z^{2}} \widehat{\mathcal{C}}_{2}\left(\frac{\mathcal{Y}_{n}}{\widehat{Q}}\right)\right)\right|_{17,10} \leq K_{3}\left|x_{0}\right|_{9,7}, \\
\left|\widehat{Q}_{1} \widehat{\mathcal{L}}^{-1}\left(\frac{\widehat{W}_{4}}{2 z^{2} \widehat{Q}^{2}} \widehat{\mathcal{C}}\left(\frac{x_{n}}{\widehat{Q}_{1}}\right) \mathcal{Y}_{n}^{2}\right)\right|_{15,10} \leq K_{4}\left|x_{0}\right|_{9,7}^{2}
\end{array}
$$


where

$$
\begin{aligned}
& K_{1}:=\pi^{2} M_{9,8}\left(1+\frac{3}{2} z^{2}\right) L_{10,8} M_{7,5}\left(\frac{\widehat{W}_{5}}{z^{5}}\right) M_{9,7}\left(\frac{z^{2}}{\widehat{Q}_{1}}\right)\left\|\cosh \left(\frac{z}{2}\right)\right\|_{7,5}, \\
& K_{2}:=\pi^{9} M_{15,8}\left(1+\frac{3}{2} z^{2}\right) L_{16,8} M_{9,1}\left(\frac{\widehat{W}_{1}}{2 z^{9} \widehat{Q}}\right) c_{9,7}, \\
& K_{3}:=\pi^{9} M_{15,8}\left(1+\frac{3}{2} z^{2}\right) L_{16,8} M_{7,-1}\left(\frac{\widehat{W}_{2}}{2 z^{11}}\right)\|\cosh (z)\|_{7,-1} M_{9,1}\left(\frac{z^{2}}{\widehat{Q}}\right) c_{9,7}, \\
& K_{4}:=\frac{1}{56 \pi^{2}} M_{13,8}\left(1+\frac{3}{2} z^{2}\right) L_{14,8} M_{14,8}\left(\frac{\widehat{W}_{4}}{2 z^{2}}\right) M_{18,12}\left(\frac{z^{4}}{\widehat{Q}^{2}}\right) \alpha_{8,8} c_{9,7}^{2} .
\end{aligned}
$$

This imply

$$
\left|x_{n+1}+\widehat{Q}_{1} \widehat{\mathcal{L}}^{-1}\left(\frac{\widehat{\mu}}{z^{2}}\right)\right|_{11,10} \leq\left(K_{1}+K_{2}+K_{3}+K_{4} K_{0}\right) K_{0}
$$

Evaluation of these quantities yields approximately

\begin{tabular}{|c|c|c|c|}
\hline$K_{1}$ & $K_{2}$ & $K_{3}$ & $K_{4}$ \\
\hline 375142.8501573 & 633.1622891589 & 10214.44411459 & 0.000011150281 \\
\hline
\end{tabular}

and altogether

$$
\left|x_{n+1}+\widehat{Q}_{1} \widehat{\mathcal{L}}^{-1}\left(\frac{\widehat{\mu}}{z^{2}}\right)\right|_{11,10} \leq 118896679183 .
$$

Now we consider $\mathrm{g}(z):=z^{2} \widehat{\mathcal{L}}^{-1}\left(\frac{\widehat{\mu}}{z^{2}}\right)$, letting $z^{2} y=z^{-2} \mathrm{~g}(z)$, with (9.15) we obtain

$$
\widehat{D} y=\widehat{D}\left(z^{-4} \mathrm{~g}\right)=f(\widehat{D})\left[\frac{\widehat{\mu}(z)\left(4+z^{2}\right)}{4 z^{4} \widehat{V}_{1}}\right]
$$

where

$$
f(z):=\frac{z}{\sinh (z / 2)}
$$

The coefficient of $z^{k}$ in $f(z)$ is smaller than $4(2 \pi)^{k}$ we obtain by comparison of the coefficients of $z^{j}$ in (9.22)

$$
(j-1)\left|\mathrm{g}_{j+3}\right| \leq 4 \sum_{k=4}^{j} *(2 \pi)^{k-j} h_{k} \frac{(j-1) !}{(k-1) !}
$$


and hence

$$
\frac{\left|\mathrm{g}_{j}\right| \pi^{j} 2^{j}}{(j-2) !} \leq 4 \sum_{k=4}^{\infty} \frac{h_{k}(2 \pi)^{k}}{(k-1) !} \leq 3668333 .
$$

We obtain for add $j \geq 9$

$$
\frac{\left|\mathrm{g}_{j}\right|}{(j-10) ! \pi^{-j}} \leq \pi^{3} \cdot 3668333 \cdot(j-9)(j-8)(j-7)(j-6)(j-5) 2^{-j+3} .
$$

and

$$
\frac{\left|g_{j-2}\right|}{(j-10) ! \pi^{-j}} \leq \pi^{5} \cdot 3668333 \cdot(j-9)(j-8)(j-7) 2^{-j+5} .
$$

but, $\left\{\frac{\widehat{Q}_{1}}{z^{2}} \mathrm{~g}(z)\right\}_{j}=\mathrm{g}_{j}+\frac{3}{2} \mathrm{~g}_{j-2}$, with (9.20) this yields eventually

$$
\frac{\left|\left\{x_{n+1}\right\}_{j}\right|}{(j-10) ! \pi^{-j}} \leq 118896679184 \text { for } j \geq 81 .
$$

This implies

$$
\frac{\left|\left\{x_{n+1}\right\}_{j}\right|}{(j-7) ! \pi^{-j}} \leq \frac{118896679184}{72 \cdot 73 \cdot 74} \leq 305691 \quad \text { for } j \geq 81
$$

and with $x_{n+1} \equiv x_{n} \bmod z^{81}$ we finally proved that $\left|x_{n+1}\right|_{9,7} \leq K_{0}=308027.35$. Thus the proof of (9.18) is complete.

\section{Theorem 9.2.}

$$
\left|\alpha_{n}\right| \leq \frac{98048.15}{(n-1)(n-2)(n-3)(n-4)(n-5)(n-6)} \quad \text { for } n \geq 9 .
$$

and even better

$$
\left|\alpha_{n}\right| \leq 37845988419 \frac{(n-10) !}{(n-1) !} \quad \text { for odd } n \geq 81 .
$$

As a consequence we obtain estimates for $\alpha$

$$
\begin{aligned}
\left|\alpha-\frac{1}{\pi} \sum_{n=1}^{N}{ }^{*} \alpha_{n}\right| & \leq 12046752267 \sum_{n=N}^{\infty} \frac{(n-10) !}{(n-1) !} \text { for } n \geq 9, \\
& \leq 12046752267 \cdot \frac{(N-10) !}{16(N-2) !}\left(1+\frac{8}{N-1}\right), \\
& \leq \frac{762333542}{N(N-1)(N-2)(N-3)(N-4)(N-5)(N-6)(N-7)}
\end{aligned}
$$

We evaluated $\alpha_{n}$ for $n=9,11, \ldots, 81$ and obtain

$$
|\alpha-1.264150331| \leq 6 \cdot 10^{-7} \text {. }
$$




\section{Références}

[1] W. Eckhaus, Asymptotic Analysis of Singular perturbations, North-Holland, Amsterdam (1979).

[2] A. Fruchard, R. Schäfke, Exponentially small splitting of separatrices for difference equations with small step size, Journal of Dynamical and Control Systems, Volume 2, Number 2 / April, 1996, 193-238.

[3] V. Hakim, K. Mallick, Exponentially small splitting of separatrices, matching in the complex plan and Borel summation, Nonlinearity 6(1993) 57-70.

[4] V.F. Lazutkin,I.G. Schachmannski and M.B. Tabanov, Splitting of separatrices for standard and semistandard mappings, Physica D, 40 235-248, (1989).

[5] R. Schäfke, H. Volkmer, Asymptotic analysis of the equichordal problem, J.reine u. Angew. Math. 425(1992), 9-60. 